\title{
Supplemental Information: Formation of Highly Oxygenated Low-Volatility Products from Cresol Oxidation
}

Rebecca H. Schwantes et al.

S1 Further Details on $\mathrm{CF}_{3} \mathrm{O}^{-}$CIMS Analysis

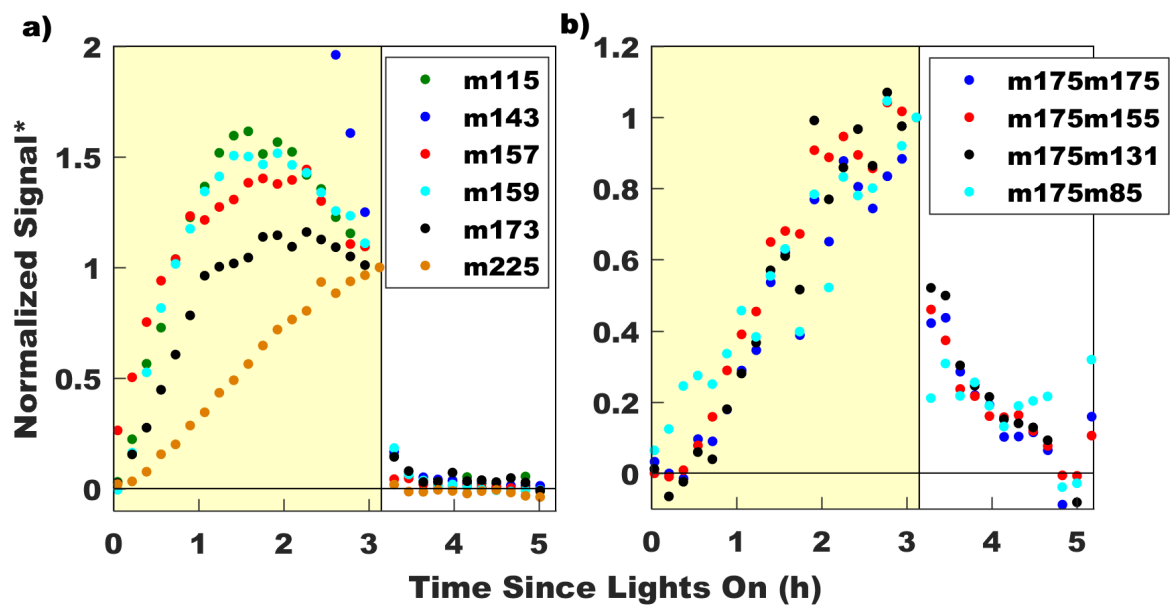

Figure S1. CIMS MS signals of 3-methyl catechol oxidation products (panel a) and MS/MS signals of tetrahydroxy toluene (panel b) for experiment 10. Desorption of compounds from instrument walls was measured by sampling photooxidation products generated in the chamber (yellow) and then immediately switching to purified air (white).*CIMS signal is normalized to time right before lights off.

Table S1: Estimated CIMS sensitivity factors

\begin{tabular}{|c|c|c|c|c|}
\hline Compound & Structure & 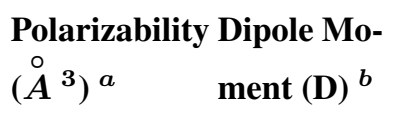 & $\begin{array}{l}\text { Sensitivity } \\
\text { Factor }^{c}\end{array}$ & Notes \\
\hline \multicolumn{5}{|c|}{ Toluene Related Compounds } \\
\hline$o$-Cresol & & 11.8 & 1 & \\
\hline
\end{tabular}


Table S1: Estimated CIMS sensitivity factors

\begin{tabular}{|c|c|c|c|c|c|}
\hline Compound & Structure & $\begin{array}{l}\text { Polarizability } \\
\left.\stackrel{\circ}{\stackrel{A}{A}^{3}}\right)^{a}\end{array}$ & $\begin{array}{l}\text { Dipole Mo- } \\
\text { ment }(D)^{b}\end{array}$ & $\begin{array}{l}\text { Sensitivity } \\
\text { Factor } c\end{array}$ & Notes \\
\hline$m$-Cresol & & 13.1 & 1.53 & 1.07 & \\
\hline$p$-Cresol & & 13 & 1.53 & 1.06 & \\
\hline 4-Methylcatechol & & 13.7 & 2.7 & 1.44 & \\
\hline $\begin{array}{l}\text { Methyl hydro- } \\
\text { quinone }\end{array}$ & & 13.7 & 2.05 & 1.21 & $\begin{array}{l}\text { Assume same polarizability as 4-methyl } \\
\text { catechol }\end{array}$ \\
\hline 3-Methylcatechol & & 13.7 & 2.42 & 1.34 & $\begin{array}{l}\text { Assume same polarizability as 4-methyl } \\
\text { catechol }\end{array}$ \\
\hline $\begin{array}{l}\text { 2-Methyl resorci- } \\
\text { nol }\end{array}$ & & 13.7 & 2 & 1.19 & $\begin{array}{l}\text { Assume same polarizability as 4-methyl } \\
\text { catechol }\end{array}$ \\
\hline $\begin{array}{l}\text { 4-Methyl resorci- } \\
\text { nol }\end{array}$ & & 13.7 & 1.81 & 1.13 & $\begin{array}{l}\text { Assume same polarizability as 4-methyl } \\
\text { catechol }\end{array}$ \\
\hline $\begin{array}{l}\text { 5-Methyl resorci- } \\
\text { nol }\end{array}$ & & 13.7 & 2.1 & 1.23 & $\begin{array}{l}\text { Assume same polarizability as } 4 \text {-methyl } \\
\text { catechol }\end{array}$ \\
\hline $\begin{array}{l}\text { 4-Methyl-2- } \\
\text { nitrophenol }\end{array}$ & & 16.2 & 3.49 & 1.69 & \\
\hline
\end{tabular}


Table S1: Estimated CIMS sensitivity factors

\begin{tabular}{lllll} 
Compound & Structure & $\begin{array}{l}\text { Polarizability Dipole Mo- } \\
\left.\stackrel{\circ}{A}^{3}\right)^{a}\end{array}$ & $\begin{array}{l}\text { Sensitivity } \\
\text { ment }(\mathbf{D})^{b}\end{array}$ & Factor $^{c}$ \\
\hline Benzoic acid & 11.3 & 1.26 & 0.92
\end{tabular}

Benzene Related Compounds

\begin{tabular}{|c|c|c|c|c|}
\hline Phenol & 11.1 & 1.54 & 1 & \\
\hline Catechol & 13.1 & 2.64 & 1.37 & \\
\hline Hydroquinone & 13.1 & 1.78 & 1.08 & Assume polarizability same as catechol. \\
\hline Resorcinol & 13.1 & 2.04 & 1.16 & Assume polarizability same as catechol. \\
\hline $\begin{array}{l}\text { 1,2,3-Benzene } \\
\text { triol }\end{array}$ & 11.1 & 3.17 & 1.47 & \\
\hline $\begin{array}{l}1,3,5 \text {-Trihydroxy } \\
\text { benzene }\end{array}$ & 11.1 & 2.7 & 1.32 & $\begin{array}{l}\text { Assume polarizability same as } 1,2,3- \\
\text { benzene triol }\end{array}$ \\
\hline$o$-Nitrophenol & 14 & 3.12 & 1.48 & \\
\hline$m$-Nitrophenol & 14 & 3.89 & 1.73 & $\begin{array}{l}\text { Assume polarizability same as } o- \\
\text { nitrophenol }\end{array}$ \\
\hline
\end{tabular}


Table S1: Estimated CIMS sensitivity factors

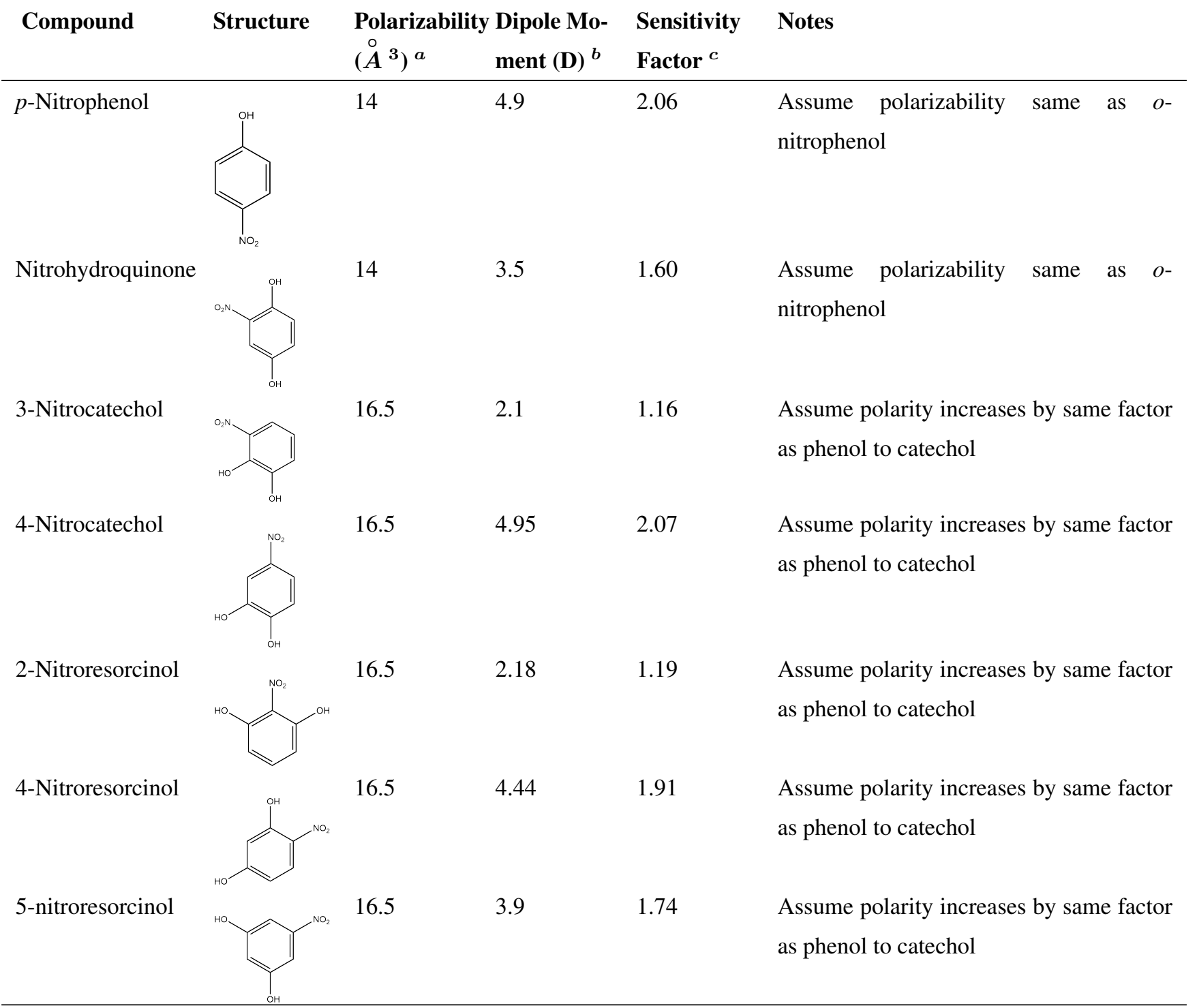

${ }^{a}$ Polarizability was estimated using the refractive index of each compound reported in Lide (2001) as done by Dewar and Stewart (1984). ${ }^{b}$ The reported dipole moment is the average of all values reported in McClellan (1974) for experiments using benzene as a solvent and taken between $20-30{ }^{\circ} \mathrm{C}$. ${ }^{c}$ The sensitivity factor equals the ion-molecule collision rate of the compound divided by the ion-molecule collision rate of $o$-cresol for toluene related compounds or phenol for benzene related compounds.

As done by Dewar and Stewart (1984), polarizability was estimated using the refractive index reported in (Lide, 2001) and the formula: $\bar{P}=(3 / 4 \pi N)(M / d)\left[\left(n^{2}-1\right) /\left(n^{2}+2\right)\right] * 10^{24}$ where $\bar{P}$ is the average polarizability, $\mathrm{n}$ is the refractive index, $\mathrm{N}$ 
Table S2. Water curve correction and sensitivity factors applied to each compound of interest

\begin{tabular}{lll}
\hline Compound & Water Curve Correction & Compound on which Sensitivity Factor is Based ${ }^{a}$ \\
\hline Cresol & $o$-cresol & Weighted $o$-, $m$-, and $p$-cresol ${ }^{b}$ \\
Dihydroxy toluene & 3-methyl catechol & 3-methyl catechol \\
Trihydroxy toluene & 3-methyl catechol & $1,2,3$ benzene triol \\
Tetrahydroxy toluene & 3-methyl catechol & $1,2,3$ benzene triol \\
Hydroxy methyl benzoquinone & $o$-cresol & $o$-cresol \\
Dihydroxy methyl benzoquinone & 3 -methyl catechol & 3-methyl catechol \\
Methyl nitrophenol & o-cresol & 4-methyl-2-nitrophenol \\
Dihydroxy nitrotoluene & 3 -methyl catechol & 3-nitrocatechol \\
Benzoic acid & $o$-cresol & benzoic acid \\
Peroxy benzoic acid & $o$-cresol & benzoic acid \\
Phenyl hydroperoxide & $o$-cresol & benzoic acid \\
Nitrosophenol & $o$-cresol & $o$-nitrophenol \\
Nitrophenol & $o$-cresol & $o$-nitrophenol \\
Dinitrophenol & $o$-cresol & $o$-nitrophenol \\
\hline
\end{tabular}

${ }^{a}$ The sensitivity factors are listed in Table S1. ${ }^{b}$ The photooxidation isomer distribution reported by Klotz et al. (1998) was used to create a generalized cresol sensitivity factor.

is Avogadro's number, $\mathrm{M}$ is the molecular weight, and $\mathrm{d}$ is the density. The dipole moments measured in benzene and reported by McClellan (1974) were used to estimate the CIMS sensitivity. Dipole moments measured in air would be more accurate than those measured in benzene. However, very few dipole moments measured in air are available for the aromatic compounds of interest. For phenol, the CIMS sensitivity decreases by $7 \%$ when using the dipole moment measured in air (Pedersen et al., 1969) versus benzene (McClellan, 1974).

As noted in Table S1 when refractive index was unavailable, the polarizability for the closest related compound was used. The ion-molecule collision rate for each compound was estimated using the polarizabilities and dipole moments reported in Table S1 and the technique described in Su and Chesnavich (1982). The sensitivity is expected to be proportional to the ion-molecule collision rate. The sensitivity factor reported in Table S1 is the ratio of the ion-molecule collision rate for the compound to that of $o$-cresol for toluene related compounds and phenol for benzene related compounds.

As stated in the main text, the $o$-cresol or 3-methyl catechol water curve was used to determine the sensitivity of a compound with a correction for the ion-molecule collision rate. In Table S2, the water curve correction and the sensitivity factor used for each compound is reported. In some cases, as specified in Table S2 the polarizability and dipole moments were not available for toluene related compounds, so the benzene counterpart was used instead. Note that depending on the fraction of isomers of dihydroxy toluene that form from $o$-cresol oxidation, dihydroxy toluene may be underestimated. 3-methyl catechol has the highest sensitivity of all the isomers that could form from $o$-cresol oxidation (3-methyl catechol, 2-methyl resorcinol, 4- 
methyl resorcionol, and methyl hydroquinone). Similarly, depending on the exact isomer distribution that forms from dihydroxy toluene oxidation, trihydroxy toluene may be underestimated. 1,3,5-trihydroxy benzene has a lower sensitivity factor (1.32) compared to that for 1,2,3 benzene triol (1.47). Polarizability and dipole moment measurements are not available for hydroxy methyl benzoquinone or dihydroxy methyl benzoquinone. Thus, we assume that hydroxy methyl benzoquinone behaves like $o$-cresol and dihydroxy methyl benzoquinone behaves like 3-methyl catechol.

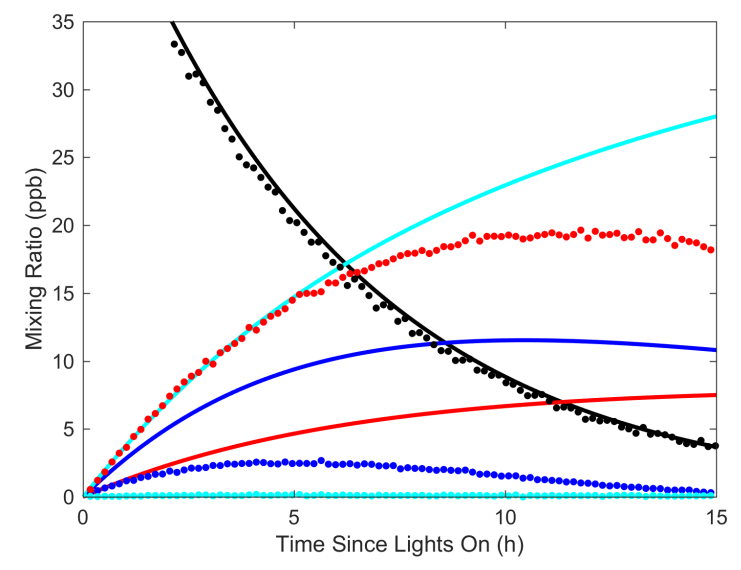

Figure S2. CIMS measurements (data points) compared to predictions from version 1 of kinetic model (lines) for benzaldehyde low-NO oxidation (experiment 10) for the following compounds benzaldehyde (black), peroxybenzoic acid (blue), benzoic acid (red), and phenyl hydroperoxide (cyan).

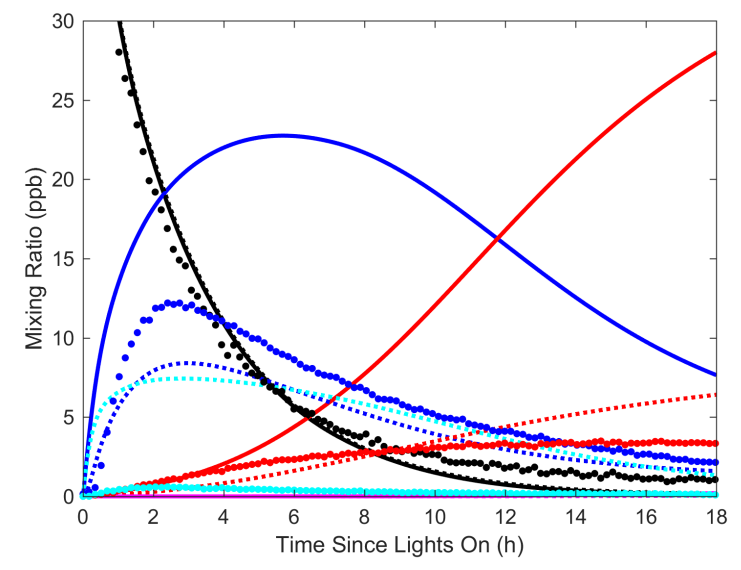

Figure S3. CIMS measurements (data points) compared to predictions from the kinetic model (solid lines version 1 and dotted lines version 3) for benzaldehyde oxidation under high-NO conditions (experiment 11) for the following compounds benzaldehyde (black), nitrophenol (blue), nitrosophenol (cyan), dinitrophenol (red), and maleic anhydride (magenta). 


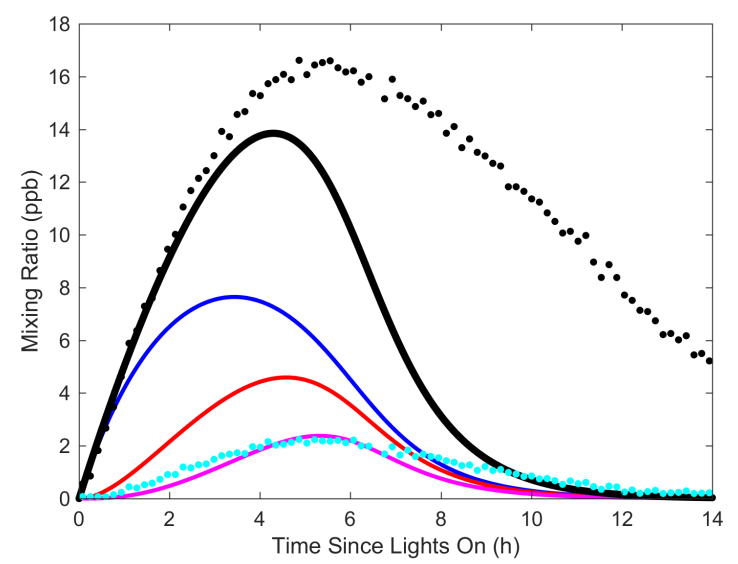

Figure S4. CIMS measurements (data points) compared to predictions from version 3 of the kinetic model (lines) for 3-methyl catechol oxidation under low-NO conditions for bicyclic intermediate products from all precursors (black), 3-methyl catechol (blue), trihydroxy toluene (red), tetrahydroxy toluene (magenta), and trihydroxy toluene or tetrahydroxy toluene tracers (cyan).

CIMS measurements and kinetic model results for products from low-NO oxidation of benzaldehyde are displayed in Figure $\mathrm{S} 2$. Benzoic acid is under-predicted by the kinetic model suggesting it is formed in a higher yield from $\mathrm{RO}_{2}+\mathrm{RO}_{2}$ reactions, $\mathrm{RO}_{2}+\mathrm{HO}_{2}$ reactions, or both. The low yield measured by the CIMS of peroxybenzoic acid, a product from only $\mathrm{RO}_{2}+\mathrm{HO}_{2}$ reaction, could be caused by a variety of factors. For example, if the $\mathrm{RO}_{2}+\mathrm{RO}_{2}$ reaction rate constant used in MCM v3.3.1 is too low, more $\mathrm{RO}_{2}+\mathrm{HO}_{2}$ reactions would occur in the kinetic model producing an over-prediction of peroxybenzoic acid. Another possibility is that the branching ratio for the $\mathrm{RO}_{2}+\mathrm{HO}_{2}$ reaction favors formation of benzoic acid more so than recommended by MCM v3.3.1. Because benzoic acid is a product from both $\mathrm{RO}_{2}+\mathrm{RO}_{2}$ and $\mathrm{RO}_{2}+\mathrm{HO}_{2}$ reactions further constraint is not possible.

Nitrosophernol is detected from benzaldehyde oxidation under high NO conditions (Figure S3). Previous studies have detected a product $\left(\mathrm{C}_{6} \mathrm{H}_{5} \mathrm{O}(\mathrm{NO})\right)$ from the reaction of phenoxy with $\mathrm{NO}$ (Tao and $\left.\mathrm{Li}, 1999\right)$. The exact isomer that forms has not been experimentally confirmed. Based on theory, nitrosophenol is the most stable isomer (Yu et al., 1995). Two kinetic studies (Berho et al., 1998; Yu et al., 1995) proposed that phenyl nitrite is the dominant isomer given that nitrosophenol, which requires rearrangement, would not form at the timescales of their studies. $\mathrm{C}_{6} \mathrm{H}_{5} \mathrm{O}(\mathrm{NO})$ was detected at the fluorine transfer at $\mathrm{m} / \mathrm{z}(-)$ 142 , implying that it is acidic like nitrosophenol. Possibly, nitrosophenol is over-predicted by version 3 of the kinetic model (Figure S3) because two isomers (nitrosophenol and phenyl nitrite) form and the CIMS is only sensitive to nitrosophenol. The reaction rate constant for $\mathrm{C}_{6} \mathrm{H}_{5} \mathrm{O}+\mathrm{NO}$ measured by Berho et al. (1998) $\left(1.65 \times 10^{-12} \mathrm{~cm}^{3}\right.$ molec $\left.^{-1} \mathrm{~s}^{-1}\right)$ is used in the revised mechanism. The reaction of $\mathrm{C}_{6} \mathrm{H}_{5} \mathrm{O}+\mathrm{NO}$ has been shown to be reversible, but not at temperatures relevant to this study (Berho et al., 1998; Yu et al., 1995).

$\mathrm{m} / \mathrm{z}$ (-) 183, assumed to be a fragment of dinitrophenol, is possibly also maleic anhydride (cluster). Maleic anhydride is 20 a decomposition product from dinitrophenol in MCM v3.3.1. However, the predicted amount of maleic anhydride formed in 
the kinetic mechanism (version 1 and 3 ) is $\sim 0.2 \mathrm{ppb}$ after 18 hours of oxidation (Figure S3). Additionally, all nitro products detected by the CIMS have a corresponding fragment at the the $\mathrm{F}^{-}$transfer minus 20 (hydroxy nitrotoluene, dihydroxy nitrotoluene, and nitrophenol). Thus, the $\mathrm{m} / \mathrm{z}(-) 183$ signal is attributed to dinitrophenol.

\section{S2 Further Details on Kinetic Model}

5 The initial conditions specified in Table 1 of the main text were used as input in the kinetic model. The kinetic model was run with 3 different versions. Version 1, the base case of the kinetic model, included reactions from MCM v3.3.1 for toluene and inorganic gas-phase chemistry and experimentally derived wall loss rates of $o$-cresol and 3-methyl catechol. Version 2 includes all reactions in Version 1 and photolysis of hydroxy nitrotoluene and dihydroxy nitrotoluene. Version 3 includes all reactions in Version 2 and oxidation products for 3-methyl catechol and benzaldehyde. The reactions and rate constants are listed in

10 Table S3 and abbreviations are defined in Table S4. These reactions were included to test the chemistry proposed in the main text. Exact branching ratios and reaction rates for these reactions are unknown. Estimates based on known reactions of similar compounds were used.

Hydrogen abstraction from the hydroxy group of 3-methyl catechol, OH3TOL, and OH4TOL is assumed to form an intermediate that then reacts with $\mathrm{NO}_{2}$ to from a nitro compound. Under low-NO conditions, there is no loss process for this intermediate in the kinetic model or MCM v3.1.1. In experiments 1 and 2, after all injections were complete, lights on was delayed for $2.5 \mathrm{~h}$ to estimate the wall loss of $o$-cresol. Wall loss of all other compounds is explained in section 4.2 .1 in the main text.

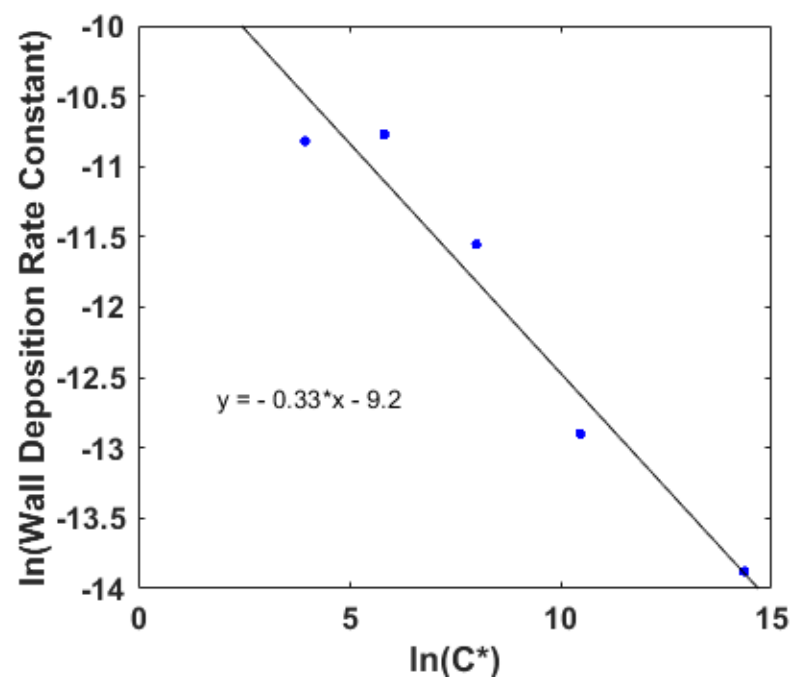

Figure S5. Linear fit to natural log of wall deposition rate constant versus natural $\log$ of $C^{*}$ used to estimate wall deposition of compounds that cannot be directly measured. 
Table S3: Reactions and reaction rate constants added to chemistry in MCM v3.3.1 to test proposed chemistry.

\begin{tabular}{|c|c|c|}
\hline New Reaction & New Reaction Rate ${ }^{a}$ & Assumptions \\
\hline \multicolumn{3}{|c|}{ Version 1 - All reactions in MCM v 3.3.1 and those listed below. } \\
\hline $\mathrm{CRESOL} \rightarrow$ wall & $9.4 \times 10^{-7} \mathrm{~s}^{-1}$ & Measured in this study \\
\hline MCATECHOL $\rightarrow$ wall & $2.5 \times 10^{-6} \mathrm{~s}^{-1}$ & Measured in this study \\
\hline \multicolumn{3}{|c|}{ Version 2 - All reactions in Version 1 and those listed below. } \\
\hline TOL1OHNO2 + hv $\rightarrow$ products & $1.73 \times 10^{-4} \mathrm{~s}^{-1}$ & Assume similar to \\
\hline MNCATECH + hv $\rightarrow$ products & $1.73 \times 10^{-4} \mathrm{~s}^{-1}$ & 6-methyl-2-nitrophenol (Bejan et al., 2007) \\
\hline \multicolumn{3}{|c|}{ Version 3 - All reactions in Version 2 and those listed below. } \\
\hline \multirow{2}{*}{$\mathrm{CRESOL}+\mathrm{OH} \rightarrow \mathrm{BCRESOL}$} & $4.65 \times 10^{-11} * 0.2 * 0.65$ & Assume missing products from \\
\hline & & Olariu et al. (2002) from bicyclic pathway. \\
\hline MCATECHOL + OH $\rightarrow$ MCATEC1O & $2.0 \times 10^{-10} * 0.07$ & Assume same as $o$-cresol \\
\hline MCATECHOL + OH $\rightarrow$ OHMBQN & $2.0 \times 10^{-10} * 0.07$ & oxidation from MCM v3.3.1 \\
\hline MCATECHOL + OH $\rightarrow$ OH3TOL & $2.0 \times 10^{-10} * 0.73$ & and (Olariu et al., 2002) \\
\hline MCATECHOL + OH $\rightarrow$ BMCATECHOL & $2.0 \times 10^{-10} * 0.13$ & \\
\hline $\mathrm{OH} 3 \mathrm{TOL}+\mathrm{OH} \rightarrow \mathrm{OH} 3 \mathrm{TOL} 1 \mathrm{O}$ & $2.5 \times 10^{-10} * 0.07$ & Assume same as $o$-cresol oxidation \\
\hline $\mathrm{OH} 3 \mathrm{TOL}+\mathrm{OH} \rightarrow \mathrm{OH} 2 \mathrm{MBQN}$ & $2.5 \times 10^{-10} * 0.07$ & from MCM v3.3.1 and (Olariu et al., 2002, 2000). \\
\hline $\mathrm{OH} 3 \mathrm{TOL}+\mathrm{OH} \rightarrow \mathrm{OH} 4 \mathrm{TOL}$ & $2.5 \times 10^{-10} * 0.73$ & Increased reaction rate constant due to additional \\
\hline $\mathrm{OH} 3 \mathrm{TOL}+\mathrm{OH} \rightarrow \mathrm{BOH} 3 \mathrm{TOL}$ & $2.5 \times 10^{-10} * 0.13$ & $\mathrm{OH}$ group to hard sphere collision rate limit. \\
\hline $\mathrm{OH} 4 \mathrm{TOL}+\mathrm{OH} \rightarrow \mathrm{OH} 4 \mathrm{TOL} 1 \mathrm{O}$ & $2.5 \times 10^{-10} * 0.07$ & Assume same as o-cresol oxidation \\
\hline $\mathrm{OH} 4 \mathrm{TOL}+\mathrm{OH} \rightarrow \mathrm{OH} 3 \mathrm{MBQN}$ & $2.5 \times 10^{-10} * 0.07$ & from MCM v3.3.1 and Olariu et al. $(2002,2000)$ \\
\hline $\mathrm{OH} 4 \mathrm{TOL}+\mathrm{OH} \rightarrow \mathrm{OH} 5 \mathrm{TOL}$ & $2.5 \times 10^{-10} * 0.73$ & Increased reaction rate constant due to additional \\
\hline $\mathrm{OH} 4 \mathrm{TOL}+\mathrm{OH} \rightarrow \mathrm{BOH} 4 \mathrm{TOL}$ & $2.5 \times 10^{-10} * 0.13$ & $\mathrm{OH}$ group to hard sphere collision rate limit. \\
\hline $\mathrm{BCRESOL}+\mathrm{OH} \rightarrow$ products & $5.44 \times 10^{-11}$ & Assume same as \\
\hline BMCATECHOL $+\mathrm{OH} \rightarrow$ products & $5.44 \times 10^{-11}$ & C5CO14OH from MCM v 3.3.1 \\
\hline $\mathrm{BOH} 3 \mathrm{TOL}+\mathrm{OH} \rightarrow$ products & $5.44 \times 10^{-11}$ & \\
\hline $\mathrm{BOH} 4 \mathrm{TOL}+\mathrm{OH} \rightarrow$ products & $5.44 \times 10^{-11}$ & \\
\hline $\mathrm{OHMBQN}+\mathrm{OH} \rightarrow$ products & $2.3 \times 10^{-11}$ & Assume same as \\
\hline $\mathrm{OH} 2 \mathrm{MBQN}+\mathrm{OH} \rightarrow$ products & $2.3 \times 10^{-11}$ & PTLQONE from MCM v 3.3.1 \\
\hline $\mathrm{OH} 3 \mathrm{TOL} 1 \mathrm{O}+\mathrm{NO}_{2} \rightarrow$ products & $2.08 \times 10^{-12}$ & Assume same as \\
\hline $\mathrm{OH} 4 \mathrm{TOL} 1 \mathrm{O}+\mathrm{NO}_{2} \rightarrow$ products & $2.08 \times 10^{-12}$ & MCATEC1O from MCM v3.3.1 \\
\hline $\mathrm{HOC} 6 \mathrm{H} 4 \mathrm{NO} 2+h v \rightarrow$ products & $6.13 \times 10^{-5} \mathrm{~s}^{-1}$ & Based on 2-nitrophenol measured \\
\hline
\end{tabular}


Table S3: Reactions and reaction rate constants added to chemistry in MCM v3.3.1 to test proposed chemistry.

\begin{tabular}{lll}
\hline New Reaction & New Reaction Rate ${ }^{a}$ & Assumptions \\
\hline $\mathrm{C} 6 \mathrm{H} 5 \mathrm{O}+\mathrm{NO} \rightarrow \mathrm{C} 6 \mathrm{H} 5 \mathrm{O}(\mathrm{NO})$ & $1.65 \times 10^{-12}$ & by Bardini (2006) reported by Chen et al. (2011) \\
\hline $\mathrm{C} 6 \mathrm{H} 5 \mathrm{O}(\mathrm{NO})+\mathrm{OH} \rightarrow$ products & $9.0 \times 10^{-13}$ & Berho et al. (1998) \\
$\mathrm{C} 6 \mathrm{H} 5 \mathrm{O}(\mathrm{NO})+\mathrm{NO}_{3} \rightarrow$ products & $9.0 \times 10^{-14}$ & HOC6H4NO2 from MCM v3.3.1 \\
\hline $\mathrm{OH} 3 \mathrm{TOL} \rightarrow$ wall & $2.1 \times 10^{-5} \mathrm{~s}^{-1}$ & Measured in this study \\
\hline $\mathrm{OH} 4 \mathrm{TOL} \rightarrow$ wall & $7.9 \times 10^{-5} \mathrm{~s}^{-1}$ & Estimated in this study \\
\hline $\mathrm{OH} 5 \mathrm{TOL} \rightarrow$ wall & $5.0 \times 10^{-4} \mathrm{~s}^{-1}$ & Estimated in this study \\
\hline $\mathrm{OHMBQN} \rightarrow$ wall & $9.6 \times 10^{-6} \mathrm{~s}^{-1}$ & Measured in this study \\
\hline $\mathrm{OH} 2 \mathrm{MBQN} \rightarrow$ wall & $2.0 \times 10^{-5} \mathrm{~s}^{-1}$ & Measured in this study \\
\hline $\mathrm{OH} 3 \mathrm{MBQN} \rightarrow$ wall & $1.2 \times 10^{-4} \mathrm{~s}^{-1}$ & Estimated in this study \\
\hline
\end{tabular}

${ }^{a}$ Reaction rate units are $\mathrm{cm}^{3}$ molec $^{-1} \mathrm{~s}^{-1}$ unless otherwise noted. 
Table S4. Abbreviations used in Table S3

\begin{tabular}{|c|c|}
\hline Abbreviation & Description \\
\hline BCRESOL & Tracer for products from the bicyclic intermediate pathway from cresol oxidation. \\
\hline BMCATECHOL & Tracer for products from the bicyclic intermediate pathway from methyl catechol. \\
\hline BOH3TOL & Tracer for products from the bicyclic intermediate pathway from trihydroxy toluene. \\
\hline BOH4TOL & Tracer for products from the bicyclic intermediate pathway from tetrahydroxy toluene. \\
\hline $\mathrm{C} 5 \mathrm{CO} 14 \mathrm{OH}$ & Acetyl acrylic acid (one of the bicyclic intermediate pathway products from o-cresol oxidation in MCM). \\
\hline CRESOL & Cresol \\
\hline HOC6H4NO2 & Nitrophenol \\
\hline MCATEC1O & Product from $\mathrm{H}$-abstraction of $\mathrm{OH}$ group of methyl catechol \\
\hline MCATECHOL & Methyl catechol \\
\hline MNCATECH & Nitro dihydroxy toluene \\
\hline $\mathrm{OH} 2 \mathrm{MBQN}$ & Dihydroxy methyl benzoquinone \\
\hline OH3MBQN & Trihydroxy methyl benzoquinone \\
\hline OH3TOL & Trihydroxy toluene \\
\hline OH3TOL1O & Product from $\mathrm{H}$-abstraction of $\mathrm{OH}$ group of trihydroxy toluene \\
\hline OH3TOL1O & Product from $\mathrm{H}$-abstraction of $\mathrm{OH}$ group of tetrahydroxy toluene \\
\hline OH4TOL & Tetrahydroxy toluene \\
\hline OH5TOL & Pentahydroxy toluene \\
\hline OHMBQN & Hydroxy methyl benzoquinone \\
\hline PTLQONE & Methyl benzoquinone (one of the bicyclic intermediate pathway products from cresol oxidation in MCM) \\
\hline TOL1OHNO2 & Nitro hydroxy toluene \\
\hline
\end{tabular}


Table S5. Chamber conditions based on kinetic model (Version 1)

\begin{tabular}{|c|c|c|c|c|c|c|c|c|c|}
\hline \multirow{2}{*}{$\begin{array}{c}\text { Expt } \\
\#\end{array}$} & \multicolumn{2}{|c|}{ VOC-OH adduct rxn (\%) } & \multicolumn{2}{|c|}{$o$-cresol rxn (\%) } & \multicolumn{2}{|c|}{ 3-methyl catechol rxn (\%) } & \multicolumn{3}{|c|}{$\mathrm{RO}_{2}$ Reaction Partner (\%) } \\
\hline & $\mathbf{O}_{2}$ & $\mathrm{NO}_{2}$ & $\mathbf{O H}$ & $\mathrm{NO}_{3}$ & $\mathbf{O H}$ & $\mathrm{NO}_{3}$ & $\mathrm{RO}_{2}$ & $\mathrm{HO}_{2}$ & NO \\
\hline 1 & 94 & 6 & $>31$ & $<69$ & $>41$ & $<59$ & $\sim 0$ & $<1$ & $>99$ \\
\hline 2 & 100 & 0 & 100 & 0 & 100 & 0 & $<12$ & $>88$ & $\sim 0$ \\
\hline 3 & 94 & 6 & $>44$ & $<56$ & $>44$ & $<56$ & $\sim 0$ & $<1$ & $>99$ \\
\hline 4 & 100 & 0 & 100 & 0 & 100 & 0 & $<6$ & $>94$ & $\sim 0$ \\
\hline 5 & $>99.9$ & $<0.1$ & $>96$ & $<4$ & $>91$ & $<9$ & $\sim 0$ & $<1$ & $>99$ \\
\hline 6 & 100 & 0 & 100 & 0 & 100 & 0 & $<1$ & $>99$ & $\sim 0$ \\
\hline 7 & $>99.9$ & $<0.1$ & NA & NA & $\sim 100$ & $\sim 0$ & $\sim 0$ & $<2$ & $>98$ \\
\hline 8 & 100 & 0 & NA & NA & 100 & 0 & \multirow{3}{*}{\multicolumn{3}{|c|}{ 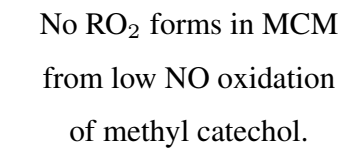 }} \\
\hline 9 & 100 & 0 & NA & NA & 100 & 0 & & & \\
\hline 10 & 100 & 0 & NA & NA & 100 & 0 & & & \\
\hline 11 & 100 & 0 & NA & NA & NA & NA & $\sim 16^{a}$ & $\sim 84^{a}$ & $\sim 0^{a}$ \\
\hline 12 & 97 & 3 & NA & NA & NA & NA & $\sim 0$ & $<4$ & $>96$ \\
\hline 13 & 100 & 0 & 100 & 0 & 100 & 0 & $<18$ & $>82$ & $\sim 0$ \\
\hline 14 & 90 & 10 & $>20$ & $<80$ & $>34$ & $<66$ & $\sim 0$ & $<1$ & $>99$ \\
\hline 15 & $>99.9$ & $<0.1$ & $>98$ & $<2$ & $>94$ & $<6$ & $\sim 0$ & $<1$ & $>99$ \\
\hline
\end{tabular}

${ }^{a}$ Throughout most of the experiment, the peroxy radical distribution was that stated. However, over the first hour there was exponential convergence to these steady state values from $\mathrm{RO}_{2}+\mathrm{RO}_{2}=100 \%$ and $\mathrm{RO}_{2}+\mathrm{HO}_{2}=0 \%$.

\section{S3 DART-MS Analysis Details and Product Identification}

\section{S3.1 DART-MS Analysis Details}

A mass calibrant and an independent quality assurance/quality control (QA/QC) compound were run with each sample set to ensure mass accuracy to within $5 \mathrm{mDa}$. The mass calibrant used for positive mode was polyethylene glycol (average molecular weight of 600 amu, PEG-600; Acros Organics, Geel, Belgium), which was dissolved in methanol. The independent QA/QC compound used is reserpine, which was purchased from Sigma-Aldrich and diluted in methanol.

Tweezers were used to introduce the samples into the DART gas stream. Before analysis, the tweezers were rinsed with acetone, and were introduced into the gas stream to vaporize any contaminants. A strip $(\sim 1 \mathrm{~cm})$ was cut from each sample substrate for testing. The cutting was tested in triplicate, with each sampling being from a different are of the substrate.

In these studies, a solution of PEG-600 (50 $\mu \mathrm{L}$ in $10 \mathrm{~mL}$ of methanol) was used to calibrate (61-679 Da) the mass spectrometer for each run. Acceptable calibration was determined if the calibration Mass Center software produced a residual value of $>9 \times 10^{-12}$. To ensure proper calibration, a solution of reserpine ( $5 \mathrm{mg}$ in $10 \mathrm{~mL}$ of methanol) was analyzed subsequent 
to the PEG-600 in every sample run. Calibration was deemed sufficient if the $\mathrm{m} / \mathrm{z}$ of reserpine fell within \pm 0.005 Da of the theoretical value $(609.281 \mathrm{Da})$.

The instrument used was a JEOL (Tokyo, Japan) AccuTOFTM mass spectrometer (JMS-T100LC) coupled with an IonSense (Saugus, MA, USA) DART® source. Ultra-pure helium was used as the ionizing gas with a flow rate of $1.75 \mathrm{~L} \mathrm{~min}^{-1}$. For

5 all analyses, the DART® source was set to a needle voltage of $\pm 3.5 \mathrm{kV}$. Electrode 1 and electrode 2 voltages were both set to $\pm 150 \mathrm{~V}$. Mass spectrometer settings include: an orifice 1 voltage of $\pm 20 \mathrm{~V}$, orifice 2 voltage of $\pm 5 \mathrm{~V}$, a ring lens voltage of \pm 5 $\mathrm{V}$, a peaks voltage of $1500 \mathrm{~V}$, a mass range of $50-1500 \mathrm{~m} / \mathrm{z}$ at 0.5 seconds per scan. A helium gas stream temperature of 325 ${ }^{\circ} \mathrm{C}$ was also employed.

\section{S3.2 DART-MS Product Identification}

10 Best available knowledge was used to assign the compounds displayed in Tables S6, S7, and S8. The smaller compounds could be fragmentation products. $\mathrm{C}_{x} \mathrm{H}_{y} \mathrm{NO}$ and $\mathrm{C}_{x} \mathrm{H}_{y} \mathrm{NO}_{2}$ were assumed to be amines. These products could also be small nitro or nitroso compounds or fragmentation products of nitrates. Products that appeared to be fragmentation products (i.e., reasonable structures could not be drawn) were excluded from the list. The structure of each compound was necessary to estimate the vapor pressure. The most probable dominant isomer was selected in all cases, but there are likely many additional structural isomers that form as well. The abundances reported in Tables S6, S7, and S8 are not meant to be used quantitatively due to uncertainties in the vapor pressure estimation methods and centroid fitting algorithm. Often each $\mathrm{m} / \mathrm{z}$ contained many over-lapping peaks and corrections were not made for isoptope effects. 
Table S6. DART-MS data from low-NO toluene oxidation (experiment 13).

\begin{tabular}{|c|c|c|c|c|c|c|c|c|c|}
\hline $\mathrm{m} / \mathrm{z}(+)(\mathrm{Da})$ & Intensity (A.U.) & $\mathrm{C}$ & $\mathrm{H}$ & $\mathrm{N}$ & $\mathrm{O}$ & $\Delta(\mathrm{mDa})^{a}$ & Smiles & Est. VP (atm) ${ }^{b}$ & Abundance $\left(\mathrm{R}_{f}\right)$ \\
\hline 69.067377 & 6671.45 & 5 & 8 & 0 & 0 & 3.05 & $\mathrm{C}=\mathrm{CC}=\mathrm{CC}$ & $6.49 \mathrm{E}-01(\mathrm{E})$ & $3.42 \mathrm{E}-12$ \\
\hline 71.046739 & 11123.71 & 4 & 6 & 0 & 1 & 2.95 & $\mathrm{CC}=\mathrm{CC}=\mathrm{O}$ & $8.30 \mathrm{E}-02(\mathrm{E})$ & $4.46 \mathrm{E}-11$ \\
\hline 71.081281 & 3477.48 & 5 & 10 & 0 & 0 & 4.79 & $\mathrm{C}=\mathrm{CCCC}$ & $6.49 \mathrm{E}-01(\mathrm{E})$ & $1.78 \mathrm{E}-12$ \\
\hline 73.064072 & 4280.44 & 4 & 8 & 0 & 1 & 1.27 & $\mathrm{CCCC}=\mathrm{O}$ & $1.27 \mathrm{E}-01(\mathrm{E})$ & $1.12 \mathrm{E}-11$ \\
\hline 75.04371 & 5382.00 & 3 & 6 & 0 & 2 & 0.89 & $\mathrm{CC}(\mathrm{CO})=\mathrm{O}$ & $6.92 \mathrm{E}-03(\mathrm{E})$ & $2.59 \mathrm{E}-10$ \\
\hline 80.048339 & 155429.63 & 5 & 5 & 1 & 0 & 1.69 & $\mathrm{C} 1=\mathrm{CC}=\mathrm{CC}=\mathrm{N} 1$ & $3.46 \mathrm{E}-02(\mathrm{~N})$ & $1.50 \mathrm{E}-09$ \\
\hline 83.082762 & 4393.36 & 6 & 10 & 0 & 0 & 3.31 & $\mathrm{C}=\mathrm{CCCC}=\mathrm{C}$ & $2.12 \mathrm{E}-01(\mathrm{E})$ & $6.89 \mathrm{E}-12$ \\
\hline 85.025484 & 4539.76 & 4 & 4 & 0 & 2 & 3.47 & $\mathrm{O}=\mathrm{CC}=\mathrm{CC}=\mathrm{O}$ & $3.42 \mathrm{E}-03(\mathrm{E})$ & $4.41 \mathrm{E}-10$ \\
\hline 87.039802 & 7748.51 & 4 & 6 & 0 & 2 & 4.80 & $\mathrm{O}=\mathrm{CCCC}=\mathrm{O}$ & $6.19 \mathrm{E}-03(\mathrm{E})$ & $4.16 \mathrm{E}-10$ \\
\hline 97.026419 & 6180.71 & 5 & 4 & 0 & 2 & 2.53 & $\mathrm{O}=\mathrm{CC} 1=\mathrm{CC}=\mathrm{CO} 1$ & $2.96 \mathrm{E}-03(\mathrm{~N})$ & $6.95 \mathrm{E}-10$ \\
\hline 97.055251 & 2871.86 & 6 & 8 & 0 & 1 & 10.09 & $\mathrm{CC}(\mathrm{C}=\mathrm{CC}=\mathrm{C})=\mathrm{O}$ & 8.87E-03 (E) & $1.08 \mathrm{E}-10$ \\
\hline 97.101391 & 2954.88 & 7 & 12 & 0 & 0 & 0.33 & $\mathrm{CC} 1 \mathrm{C}=\mathrm{CCCC} 1$ & $6.93 \mathrm{E}-02(\mathrm{E})$ & $1.42 \mathrm{E}-11$ \\
\hline 99.043366 & 9545.71 & 5 & 6 & 0 & 2 & 1.24 & $\mathrm{O}=\mathrm{C}(\mathrm{C}) \mathrm{C}=\mathrm{CC}=\mathrm{O}$ & $1.12 \mathrm{E}-03(\mathrm{E})$ & 2.84E-09 \\
\hline 99.072496 & 3758.17 & 6 & 10 & 0 & 1 & 8.49 & $\mathrm{CC}(\mathrm{C}=\mathrm{CCC})=\mathrm{O}$ & 8.87E-03 (E) & $1.41 \mathrm{E}-10$ \\
\hline 101.057523 & 4501.87 & 5 & 8 & 0 & 2 & 2.73 & $\mathrm{O}=\mathrm{C}(\mathrm{C}) \mathrm{CCC}=\mathrm{O}$ & $2.02 \mathrm{E}-03(\mathrm{E})$ & $7.40 \mathrm{E}-10$ \\
\hline 109.035545 & 3507.03 & 6 & 4 & 0 & 2 & -6.59 & $\mathrm{O}=\mathrm{C} 1 \mathrm{C}=\mathrm{CC}(\mathrm{C}=\mathrm{C} 1)=\mathrm{O}$ & $2.48 \mathrm{E}-05(\mathrm{E})$ & 4.69E-08 \\
\hline 109.096678 & 4467.03 & 8 & 12 & 0 & 0 & 5.05 & $\mathrm{C}=\mathrm{CC}=\mathrm{CC}=\mathrm{CCC}$ & $2.27 \mathrm{E}-02(\mathrm{E})$ & $6.55 \mathrm{E}-11$ \\
\hline 111.043476 & 6721.86 & 6 & 6 & 0 & 2 & 1.13 & $\mathrm{O}=\mathrm{CC} 1=\mathrm{CC}=\mathrm{C}(\mathrm{O} 1) \mathrm{C}$ & $1.25 \mathrm{E}-03(\mathrm{~N})$ & $1.79 \mathrm{E}-09$ \\
\hline 111.11751 & 3129.72 & 8 & 14 & 0 & 0 & -0.13 & $\mathrm{CCC}=\mathrm{CC}=\mathrm{CCC}$ & $2.27 \mathrm{E}-02(\mathrm{E})$ & $4.59 \mathrm{E}-11$ \\
\hline 113.019938 & 3471.30 & 5 & 4 & 0 & 3 & 3.93 & $\mathrm{O}=\mathrm{C} 1 \mathrm{C}(\mathrm{C})=\mathrm{CC}(\mathrm{O} 1)=\mathrm{O}$ & $7.03 \mathrm{E}-04(\mathrm{E})$ & $1.64 \mathrm{E}-09$ \\
\hline 113.05728 & 4021.97 & 6 & 8 & 0 & 2 & 2.97 & $\mathrm{O}=\mathrm{CCCC}=\mathrm{CC}=\mathrm{O}$ & $4.92 \mathrm{E}-04(\mathrm{E})$ & $2.72 \mathrm{E}-09$ \\
\hline 115.038947 & 6483.21 & 5 & 6 & 0 & 3 & 0.57 & $\mathrm{O}=\mathrm{C}(\mathrm{C}) \mathrm{C}=\mathrm{CC}(\mathrm{O})=\mathrm{O}$ & $6.57 \mathrm{E}-06(\mathrm{E})$ & $3.28 \mathrm{E}-07$ \\
\hline 115.064062 & 2955.96 & 6 & 10 & 0 & 2 & 11.84 & $\mathrm{O}=\mathrm{CCCCCC}=\mathrm{O}$ & $6.62 \mathrm{E}-04(\mathrm{E})$ & $1.49 \mathrm{E}-09$ \\
\hline 127.039667 & 5945.22 & 6 & 6 & 0 & 3 & -0.15 & $\mathrm{O}=\mathrm{C}(\mathrm{C}) \mathrm{C}=\mathrm{CC}(\mathrm{C}=\mathrm{O})=\mathrm{O}$ & $1.64 \mathrm{E}-04(\mathrm{E})$ & $1.21 \mathrm{E}-08$ \\
\hline 127.112254 & 4155.67 & 8 & 14 & 0 & 1 & 0.04 & $\mathrm{O}=\mathrm{CCCCCC}=\mathrm{CC}$ & $1.45 \mathrm{E}-03(\mathrm{E})$ & $9.55 \mathrm{E}-10$ \\
\hline 129.053327 & 4254.59 & 6 & 8 & 0 & 3 & 1.84 & $\mathrm{O}=\mathrm{C}(\mathrm{C}=\mathrm{CC}(\mathrm{O}) \mathrm{C}=\mathrm{O}) \mathrm{C}$ & $6.36 \mathrm{E}-05(\mathrm{E})$ & $2.22 \mathrm{E}-08$ \\
\hline 139.034538 & 5275.37 & 7 & 6 & 0 & 3 & 4.98 & $\mathrm{CC} 1=\mathrm{CC}(\mathrm{C}=\mathrm{C}(\mathrm{O}) \mathrm{C} 1=\mathrm{O})=\mathrm{O}$ & $5.26 \mathrm{E}-07(\mathrm{E})$ & $3.34 \mathrm{E}-06$ \\
\hline 141.050361 & 3122.55 & 7 & 8 & 0 & 3 & 4.81 & $\mathrm{CC} 1=\mathrm{CC}=\mathrm{C}(\mathrm{O}) \mathrm{C}(\mathrm{O})=\mathrm{C} 1 \mathrm{O}$ & $5.97 \mathrm{E}-08(\mathrm{~N})$ & $1.74 \mathrm{E}-05$ \\
\hline 155.034837 & 3399.08 & 7 & 6 & 0 & 4 & -0.40 & $\mathrm{CC} 1=\mathrm{CC}(\mathrm{C}(\mathrm{O})=\mathrm{C}(\mathrm{O}) \mathrm{C} 1=\mathrm{O})=\mathrm{O}$ & $8.26 \mathrm{E}-09(\mathrm{E})$ & $1.37 \mathrm{E}-04$ \\
\hline 157.045695 & 8025.67 & 7 & 8 & 0 & 4 & 4.39 & $\mathrm{CC} 1=\mathrm{CC}(\mathrm{O})=\mathrm{C}(\mathrm{O}) \mathrm{C}(\mathrm{O})=\mathrm{C} 1 \mathrm{O}$ & $3.28 \mathrm{E}-10(\mathrm{~N})$ & $8.13 \mathrm{E}-03$ \\
\hline 161.091424 & 2838.46 & 11 & 12 & 0 & 1 & 5.22 & $\mathrm{O}=\mathrm{CC}=\mathrm{CC}=\mathrm{CC}=\mathrm{CC}=\mathrm{CC}=\mathrm{C}$ & $3.32 \mathrm{E}-05(\mathrm{E})$ & $2.85 \mathrm{E}-08$ \\
\hline 173.044149 & 3338.73 & 7 & 8 & 0 & 5 & 0.85 & $\mathrm{CC} 1=\mathrm{C}(\mathrm{O}) \mathrm{C}(\mathrm{O})=\mathrm{C}(\mathrm{C}(\mathrm{O})=\mathrm{C} 1 \mathrm{O}) \mathrm{O}$ & $1.12 \mathrm{E}-12(\mathrm{~N})$ & $9.92 \mathrm{E}-01$ \\
\hline 203.10019 & 3898.22 & 13 & 14 & 0 & 2 & 7.01 & $\mathrm{O}=\mathrm{CC}=\mathrm{CC}=\mathrm{CC}=\mathrm{CC}=\mathrm{CC}=\mathrm{CC}(\mathrm{C})=\mathrm{O}$ & $1.46 \mathrm{E}-07(\mathrm{E})$ & $8.87 \mathrm{E}-06$ \\
\hline
\end{tabular}

\footnotetext{
${ }^{a}$ The difference between the measured and proposed compound exact mass. ${ }^{b}$ Est. VP = Estimated vapor pressure. Estimation Method in parenthesis: $\mathrm{E}=\mathrm{EVAPORATION}$ method and $\mathrm{N}=$ Nannoolal method.
} 
Table S7: DART-MS data from high-NO $o$-cresol oxidation (experiment 15).

\begin{tabular}{|c|c|c|c|c|c|c|c|c|c|}
\hline m/z (+) (Da) & Intensity $^{a}$ & $\mathrm{C}$ & $\mathrm{H}$ & $\mathrm{N}$ & $\mathrm{O}$ & $\Delta^{b}(\mathrm{mDa})$ & Smiles & Est. VP $(\mathrm{atm})^{c}$ & Abundance $\left(\mathrm{R}_{f}\right)$ \\
\hline 69.06738 & 4799.51 & 5 & 8 & 0 & 0 & 3.05 & $\mathrm{C}=\mathrm{CC}=\mathrm{CC}$ & 6.49E-01 (E) & $1.17 \mathrm{E}-11$ \\
\hline 71.04674 & 7360.97 & 4 & 6 & 0 & 1 & 2.95 & $\mathrm{CC}=\mathrm{CC}=\mathrm{O}$ & 8.30E-02 (E) & $1.40 \mathrm{E}-10$ \\
\hline 73.06407 & 3055.72 & 4 & 8 & 0 & 1 & 1.27 & $\mathrm{CCCC}=\mathrm{O}$ & $1.27 \mathrm{E}-01(\mathrm{E})$ & $3.82 \mathrm{E}-11$ \\
\hline 75.04371 & 3495.06 & 3 & 6 & 0 & 2 & 0.89 & $\mathrm{CC}(\mathrm{CO})=\mathrm{O}$ & $6.92 \mathrm{E}-03$ (E) & 7.99E-10 \\
\hline 76.0358 & 2830.61 & 2 & 5 & 1 & 2 & 4.06 & $\mathrm{OCC}(\mathrm{N})=\mathrm{O}$ & $5.43 \mathrm{E}-08(\mathrm{~N})$ & $8.25 \mathrm{E}-05$ \\
\hline 80.04834 & 2555.95 & 5 & 5 & 1 & 0 & 1.69 & $\mathrm{C} 1=\mathrm{CC}=\mathrm{CC}=\mathrm{N} 1$ & $3.46 \mathrm{E}-02(\mathrm{~N})$ & $1.17 \mathrm{E}-10$ \\
\hline 81.0676 & 2116.91 & 6 & 8 & 0 & 0 & 2.83 & $\mathrm{C}=\mathrm{CC}=\mathrm{CC}=\mathrm{C}$ & 2.12E-01 (E) & $1.58 \mathrm{E}-11$ \\
\hline 83.08276 & 2977.45 & 6 & 10 & 0 & 0 & 3.31 & $\mathrm{C}=\mathrm{CCCC}=\mathrm{C}$ & 2.12E-01 (E) & $2.22 \mathrm{E}-11$ \\
\hline 85.02548 & 2862.51 & 4 & 4 & 0 & 2 & 3.47 & $\mathrm{O}=\mathrm{CC}=\mathrm{CC}=\mathrm{O}$ & 3.42E-03 (E) & 1.32E-09 \\
\hline 85.06327 & 2015.80 & 5 & 8 & 0 & 1 & 2.07 & $\mathrm{CC}(\mathrm{C}=\mathrm{CC})=\mathrm{O}$ & 2.71E-02 (E) & $1.17 \mathrm{E}-10$ \\
\hline 87.04526 & 4217.44 & 4 & 6 & 0 & 2 & -0.66 & $\mathrm{O}=\mathrm{CCCC}=\mathrm{O}$ & $6.19 \mathrm{E}-03$ (E) & $1.08 \mathrm{E}-09$ \\
\hline 94.06261 & 2373.32 & 6 & 7 & 1 & 0 & 3.06 & $\mathrm{~N} 1 \mathrm{C}=\mathrm{CC}=\mathrm{CC}=\mathrm{C} 1$ & $2.04 \mathrm{E}-02(\mathrm{~N})$ & $1.84 \mathrm{E}-10$ \\
\hline 95.08159 & 1934.09 & 7 & 10 & 0 & 0 & 4.49 & $\mathrm{CC} 1 \mathrm{C}=\mathrm{CCC}=\mathrm{C} 1$ & 6.93E-02 (E) & $4.41 \mathrm{E}-11$ \\
\hline 97.02642 & 4443.40 & 5 & 4 & 0 & 2 & 2.53 & $\mathrm{O}=\mathrm{CC} 1=\mathrm{CC}=\mathrm{CO} 1$ & $2.96 \mathrm{E}-03(\mathrm{~N})$ & $2.38 \mathrm{E}-09$ \\
\hline 97.06102 & 3408.60 & 6 & 8 & 0 & 1 & 4.32 & $\mathrm{CC}(\mathrm{C}=\mathrm{CC}=\mathrm{C})=\mathrm{O}$ & 8.87E-03 (E) & $6.08 \mathrm{E}-10$ \\
\hline 97.09562 & 2367.86 & 7 & 12 & 0 & 0 & 6.10 & $\mathrm{CC} 1 \mathrm{C}=\mathrm{CCCC} 1$ & 6.93E-02 (E) & $5.40 \mathrm{E}-11$ \\
\hline 98.06128 & 2115.28 & 5 & 7 & 1 & 1 & -0.69 & $\mathrm{NC}(\mathrm{C}=\mathrm{CC}=\mathrm{C})=\mathrm{O}$ & $1.44 \mathrm{E}-06(\mathrm{~N})$ & 2.33E-06 \\
\hline 99.04337 & 5939.65 & 5 & 6 & 0 & 2 & 1.24 & $\mathrm{O}=\mathrm{C}(\mathrm{C}) \mathrm{C}=\mathrm{CC}=\mathrm{O}$ & $1.12 \mathrm{E}-03(\mathrm{E})$ & 8.40E-09 \\
\hline 99.07832 & 3689.73 & 6 & 10 & 0 & 1 & 2.67 & $\mathrm{CC}(\mathrm{C}=\mathrm{CCC})=\mathrm{O}$ & 8.87E-03 (E) & $6.58 \mathrm{E}-10$ \\
\hline 100.0362 & 2821.45 & 4 & 5 & 1 & 2 & 3.65 & $\mathrm{O}=\mathrm{CC}=\mathrm{CC}(\mathrm{N})=\mathrm{O}$ & $2.02 \mathrm{E}-07(\mathrm{~N})$ & $2.21 \mathrm{E}-05$ \\
\hline 101.0222 & 2194.10 & 4 & 4 & 0 & 3 & 1.65 & $\mathrm{O}=\mathrm{CC}=\mathrm{CC}(\mathrm{O})=\mathrm{O}$ & 2.01E-05 (E) & $1.73 \mathrm{E}-07$ \\
\hline 101.0575 & 3823.68 & 5 & 8 & 0 & 2 & 2.73 & $\mathrm{O}=\mathrm{C}(\mathrm{C}) \mathrm{CCC}=\mathrm{O}$ & 2.02E-03 (E) & 2.99E-09 \\
\hline 102.0545 & 2971.25 & 4 & 7 & 1 & 2 & 1.54 & $\mathrm{NCC}=\mathrm{CC}(\mathrm{O})=\mathrm{O}$ & $2.92 \mathrm{E}-05(\mathrm{~N})$ & $1.61 \mathrm{E}-07$ \\
\hline 104.0332 & 5611.42 & 3 & 5 & 1 & 3 & 1.60 & $\mathrm{CC}=\mathrm{CON}(=\mathrm{O})=\mathrm{O}$ & 2.60E-02 (E) & $3.42 \mathrm{E}-10$ \\
\hline 109.1028 & 3360.34 & 8 & 12 & 0 & 0 & -1.07 & $\mathrm{C}=\mathrm{CC}=\mathrm{CC}=\mathrm{CCC}$ & 2.27E-02 (E) & $2.34 \mathrm{E}-10$ \\
\hline 111.0435 & 4461.87 & 6 & 6 & 0 & 2 & 1.13 & $\mathrm{O}=\mathrm{CC} 1=\mathrm{CC}=\mathrm{C}(\mathrm{O} 1) \mathrm{C}$ & $1.25 \mathrm{E}-03(\mathrm{~N})$ & 5.64E-09 \\
\hline 111.1175 & 2507.94 & 8 & 14 & 0 & 0 & -0.13 & $\mathrm{CCC}=\mathrm{CC}=\mathrm{CCC}$ & 2.27E-02 (E) & $1.75 \mathrm{E}-10$ \\
\hline 113.0262 & 1862.29 & 5 & 4 & 0 & 3 & -2.29 & $\mathrm{O}=\mathrm{C} 1 \mathrm{C}(\mathrm{C})=\mathrm{CC}(\mathrm{O} 1)=\mathrm{O}$ & 7.03E-04 (E) & 4.19E-09 \\
\hline 113.0573 & 3145.91 & 6 & 8 & 0 & 2 & 2.97 & $\mathrm{O}=\mathrm{CCCC}=\mathrm{CC}=\mathrm{O}$ & 4.92E-04 (E) & $1.01 \mathrm{E}-08$ \\
\hline 114.0553 & 2382.57 & 5 & 7 & 1 & 2 & 0.17 & $\mathrm{C}=\mathrm{C}(\mathrm{C}=\mathrm{CC}(\mathrm{O})=\mathrm{O}) \mathrm{N}$ & $1.45 \mathrm{E}-05(\mathrm{~N})$ & $2.59 \mathrm{E}-07$ \\
\hline 115.0389 & 3284.56 & 5 & 6 & 0 & 3 & 0.57 & $\mathrm{O}=\mathrm{C}(\mathrm{C}) \mathrm{C}=\mathrm{CC}(\mathrm{O})=\mathrm{O}$ & $6.57 \mathrm{E}-06(\mathrm{E})$ & 7.90E-07 \\
\hline 115.0703 & 2005.33 & 6 & 10 & 0 & 2 & 5.56 & $\mathrm{O}=\mathrm{CCCCCC}=\mathrm{O}$ & 6.62E-04 (E) & 4.79E-09 \\
\hline 118.0469 & 2527.17 & 4 & 7 & 1 & 3 & 3.51 & $\mathrm{CCC}=\mathrm{CON}(=\mathrm{O})=\mathrm{O}$ & $8.50 \mathrm{E}-03$ (E) & 4.71E-10 \\
\hline 120.0524 & 3185.49 & 4 & 9 & 1 & 3 & 13.66 & $\operatorname{CCCCON}(=\mathrm{O})=\mathrm{O}$ & $8.50 \mathrm{E}-03(\mathrm{E})$ & 5.93E-10 \\
\hline 126.0519 & 1896.52 & 6 & 7 & 1 & 2 & 3.58 & $\mathrm{OC} 1=\mathrm{CN}=\mathrm{C}(\mathrm{C}) \mathrm{C}(\mathrm{O})=\mathrm{C} 1$ & $1.36 \mathrm{E}-05(\mathrm{~N})$ & $2.20 \mathrm{E}-07$ \\
\hline
\end{tabular}


Table S7: DART-MS data from high-NO $o$-cresol oxidation (experiment 15).

\begin{tabular}{|c|c|c|c|c|c|c|c|c|c|}
\hline $\mathrm{m} / \mathrm{z}(+)(\mathrm{Da})$ & Intensity $^{a}$ & $\mathrm{C}$ & $\mathrm{H}$ & $\mathrm{N}$ & $\mathrm{O}$ & $\Delta^{b}(\mathrm{mDa})$ & Smiles & Est. VP $(\mathrm{atm})^{c}$ & Abundance $\left(\mathrm{R}_{f}\right)$ \\
\hline 127.0397 & 3814.74 & 6 & 6 & 0 & 3 & -0.15 & $\mathrm{O}=\mathrm{C}(\mathrm{C}) \mathrm{C}=\mathrm{CC}(\mathrm{C}=\mathrm{O})=\mathrm{O}$ & $1.64 \mathrm{E}-04(\mathrm{E})$ & $3.68 \mathrm{E}-08$ \\
\hline 127.0661 & 2199.51 & 7 & 10 & 0 & 2 & 9.84 & $\mathrm{O}=\mathrm{C}(\mathrm{C}) \mathrm{CC}=\mathrm{CCC}=\mathrm{O}$ & 2.16E-04 (E) & $1.61 \mathrm{E}-08$ \\
\hline 127.1123 & 3306.85 & 8 & 14 & 0 & 1 & 0.04 & $\mathrm{O}=\mathrm{CCCCCC}=\mathrm{CC}$ & $1.45 \mathrm{E}-03(\mathrm{E})$ & 3.61E-09 \\
\hline 128.071 & 1864.39 & 6 & 9 & 1 & 2 & 0.14 & $\mathrm{O}=\mathrm{C}(\mathrm{C}=\mathrm{CC}=\mathrm{CCN}) \mathrm{O}$ & $3.46 \mathrm{E}-06(\mathrm{~N})$ & $8.52 \mathrm{E}-07$ \\
\hline 129.0533 & 2463.10 & 6 & 8 & 0 & 3 & 1.84 & $\mathrm{O}=\mathrm{C}(\mathrm{C}=\mathrm{CC}(\mathrm{O}) \mathrm{C}=\mathrm{O}) \mathrm{C}$ & 6.36E-05 (E) & $6.12 \mathrm{E}-08$ \\
\hline 130.0527 & 1871.23 & 5 & 7 & 1 & 3 & -2.32 & $\mathrm{O}=\mathrm{N}(\mathrm{OC}=\mathrm{CC}=\mathrm{CC})=\mathrm{O}$ & $2.78 \mathrm{E}-03(\mathrm{E})$ & $1.07 \mathrm{E}-09$ \\
\hline 139.0414 & 2114.31 & 7 & 6 & 0 & 3 & -1.92 & $\mathrm{CC} 1=\mathrm{CC}(\mathrm{C}=\mathrm{C}(\mathrm{O}) \mathrm{C} 1=\mathrm{O})=\mathrm{O}$ & $5.26 \mathrm{E}-07(\mathrm{E})$ & $6.36 \mathrm{E}-06$ \\
\hline 142.0463 & 2154.17 & 6 & 7 & 1 & 3 & 4.15 & $\mathrm{C}=\mathrm{CC}=\mathrm{CC}(\mathrm{ON}(=\mathrm{O})=\mathrm{O})=\mathrm{C}$ & $9.08 \mathrm{E}-04(\mathrm{E})$ & $3.75 \mathrm{E}-09$ \\
\hline 154.0524 & 1956.21 & 7 & 7 & 1 & 3 & -2.01 & $\begin{array}{l}\mathrm{OC} 1=\mathrm{C}(\mathrm{N}(=\mathrm{O})=\mathrm{O}) \mathrm{C}=\mathrm{CC} \\
=\mathrm{C} 1 \mathrm{C}\end{array}$ & $1.77 \mathrm{E}-05(\mathrm{~N})$ & $1.75 \mathrm{E}-07$ \\
\hline 155.0348 & 3607.35 & 7 & 6 & 0 & 4 & -0.40 & $\begin{array}{l}\mathrm{CC} 1=\mathrm{CC}(\mathrm{C}(\mathrm{O})=\mathrm{C}(\mathrm{O}) \mathrm{C} 1=\mathrm{O}) \\
=\mathrm{O}\end{array}$ & 8.26E-09 (E) & $6.91 \mathrm{E}-04$ \\
\hline 157.0457 & 1941.16 & 7 & 8 & 0 & 4 & 4.39 & $\begin{array}{l}\mathrm{CC} 1=\mathrm{CC}(\mathrm{O})=\mathrm{C}(\mathrm{O}) \mathrm{C}(\mathrm{O}) \\
=\mathrm{C} 1 \mathrm{O}\end{array}$ & $3.28 \mathrm{E}-10(\mathrm{~N})$ & $9.36 \mathrm{E}-03$ \\
\hline 267.1658 & 2870.33 & 15 & 22 & 0 & 4 & -6.13 & $\begin{array}{l}\mathrm{OC}(\mathrm{OC} 1=\mathrm{C}(\mathrm{O}) \mathrm{C}(\mathrm{O}) \\
=\mathrm{CC}=\mathrm{C} 1 \mathrm{C}) \mathrm{CCCC}=\mathrm{CC}^{c}\end{array}$ & $4.59 \mathrm{E}-12(\mathrm{~N})$ & $9.90 \mathrm{E}-01$ \\
\hline
\end{tabular}

${ }^{a}$ (A.U.) ${ }^{b}$ The difference between the measured and proposed compound exact mass. ${ }^{c}$ Est. VP $=$ Estimated vapor pressure. Estimation Method in parenthesis: $\mathrm{E}=$ EVAPORATION method, and $\mathrm{N}=$ Nannoolal method. ${ }^{c}$ Smiles in table is that of the structure predicted to form. Vapor pressure method could not estimate the vapor pressure of this structure so a very similar structure was used instead $(\mathrm{OC}(\mathrm{C}(\mathrm{O})=\mathrm{CC}=\mathrm{C} 1 \mathrm{C})=\mathrm{C} 1 \mathrm{OCC}(\mathrm{O}) \mathrm{CCCC}=\mathrm{CC})$.

Table S8: DART-MS data from high-NO toluene oxidation (experiment 14).

\begin{tabular}{ccccccclcc}
\hline $\mathrm{m} / \mathrm{z}(+)(\mathrm{Da})$ & Intensity $^{a}$ & $\mathrm{C}$ & $\mathrm{H}$ & $\mathrm{N}$ & $\mathrm{O}$ & $\Delta^{b}(\mathrm{mDa})$ & Smiles & Est. VP $(\mathrm{atm}){ }^{c}$ & Abundance $\left(\mathrm{R}_{f}\right)$ \\
\hline 61.026497 & 5540.57 & 2 & 4 & 0 & 2 & 2.46 & $\mathrm{CC}(\mathrm{O})=\mathrm{O}$ & $4.49 \mathrm{E}-03(\mathrm{E})$ & $7.02 \mathrm{E}-10$ \\
69.067377 & 1410.70 & 5 & 8 & 0 & 0 & 3.05 & $\mathrm{C}=\mathrm{CC}=\mathrm{CC}$ & $6.49 \mathrm{E}-01(\mathrm{E})$ & $1.24 \mathrm{E}-12$ \\
76.035796 & 3623.11 & 2 & 5 & 1 & 2 & 4.06 & $\mathrm{OCC}(\mathrm{N})=\mathrm{O}$ & $5.43 \mathrm{E}-08(\mathrm{~N})$ & $3.80 \mathrm{E}-05$ \\
80.048339 & 1641.73 & 5 & 5 & 1 & 0 & 1.69 & $\mathrm{C} 1=\mathrm{CC}=\mathrm{CC}=\mathrm{N} 1$ & $3.46 \mathrm{E}-02(\mathrm{~N})$ & $2.71 \mathrm{E}-11$ \\
83.082762 & 1684.25 & 6 & 10 & 0 & 0 & 3.31 & $\mathrm{C}=\mathrm{CCCC}=\mathrm{C}$ & $2.12 \mathrm{E}-01(\mathrm{E})$ & $4.52 \mathrm{E}-12$ \\
85.025484 & 2836.08 & 4 & 4 & 0 & 2 & 3.47 & $\mathrm{O}=\mathrm{CC}=\mathrm{CC}=\mathrm{O}$ & $3.42 \mathrm{E}-03(\mathrm{E})$ & $4.72 \mathrm{E}-10$ \\
87.007038 & 2179.84 & 3 & 2 & 0 & 3 & 1.18 & $\mathrm{O}=\mathrm{CC}(\mathrm{C}=\mathrm{O})=\mathrm{O}$ & $7.03 \mathrm{E}-02(\mathrm{E})$ & $1.77 \mathrm{E}-11$ \\
87.039802 & 6440.08 & 4 & 6 & 0 & 2 & 4.80 & $\mathrm{O}=\mathrm{CCCC}=\mathrm{O}$ & $6.19 \mathrm{E}-03(\mathrm{E})$ & $5.93 \mathrm{E}-10$ \\
90.013837 & 2080.44 & 2 & 3 & 1 & 3 & 5.28 & $\mathrm{C}=\mathrm{CON}(=\mathrm{O})=\mathrm{O}$ & $7.95 \mathrm{E}-02(\mathrm{E})$ & $1.49 \mathrm{E}-11$ \\
90.047162 & 1148.22 & 3 & 7 & 1 & 2 & 8.34 & $\mathrm{OC}(\mathrm{C}) \mathrm{C}(\mathrm{N})=\mathrm{O}$ & $2.13 \mathrm{E}-07(\mathrm{~N})$ & $3.07 \mathrm{E}-06$ \\
94.06261 & 7800.17 & 6 & 7 & 1 & 0 & 3.06 & $\mathrm{~N} 1 \mathrm{C}=\mathrm{CC}=\mathrm{CC}=\mathrm{C} 1$ & $2.04 \mathrm{E}-02(\mathrm{~N})$ & $2.18 \mathrm{E}-10$
\end{tabular}


Table S8: DART-MS data from high-NO toluene oxidation (experiment 14).

\begin{tabular}{|c|c|c|c|c|c|c|c|c|c|}
\hline $\mathrm{m} / \mathrm{z}(+)(\mathrm{Da})$ & Intensity $^{a}$ & $\mathrm{C}$ & $\mathrm{H}$ & $\mathrm{N}$ & $\mathrm{O}$ & $\Delta^{b}(\mathrm{mDa})$ & Smiles & Est. VP $(\mathrm{atm})^{c}$ & Abundance $\left(\mathrm{R}_{f}\right)$ \\
\hline 95.053048 & 1261.49 & 6 & 6 & 0 & 1 & -3.36 & $\mathrm{OC} 1=\mathrm{CC}=\mathrm{CC}=\mathrm{C} 1$ & $1.44 \mathrm{E}-03(\mathrm{~N})$ & $4.98 \mathrm{E}-10$ \\
\hline 95.081585 & 1364.08 & 7 & 10 & 0 & 0 & 4.49 & $\mathrm{CC} 1 \mathrm{C}=\mathrm{CCC}=\mathrm{C} 1$ & 6.93E-02 (E) & $1.12 \mathrm{E}-11$ \\
\hline 97.026419 & 6227.32 & 5 & 4 & 0 & 2 & 2.53 & $\mathrm{O}=\mathrm{CC} 1=\mathrm{CC}=\mathrm{CO} 1$ & $2.96 \mathrm{E}-03(\mathrm{~N})$ & $1.20 \mathrm{E}-09$ \\
\hline 97.061018 & 4637.55 & 6 & 8 & 0 & 1 & 4.32 & $\mathrm{CC}(\mathrm{C}=\mathrm{CC}=\mathrm{C})=\mathrm{O}$ & 8.87E-03 (E) & $2.98 \mathrm{E}-10$ \\
\hline 98.06128 & 1349.16 & 5 & 7 & 1 & 1 & -0.69 & $\mathrm{NC}(\mathrm{C}=\mathrm{CC}=\mathrm{C})=\mathrm{O}$ & $1.44 \mathrm{E}-06(\mathrm{~N})$ & 5.35E-07 \\
\hline 99.043366 & 11466.81 & 5 & 6 & 0 & 2 & 1.24 & $\mathrm{O}=\mathrm{C}(\mathrm{C}) \mathrm{C}=\mathrm{CC}=\mathrm{O}$ & $1.12 \mathrm{E}-03(\mathrm{E})$ & 5.84E-09 \\
\hline 99.089977 & 2449.13 & 6 & 10 & 0 & 1 & -8.99 & $\mathrm{CC}(\mathrm{C}=\mathrm{CCC})=\mathrm{O}$ & 8.87E-03 (E) & $1.57 \mathrm{E}-10$ \\
\hline 100.042055 & 2453.04 & 4 & 5 & 1 & 2 & -2.20 & $\mathrm{O}=\mathrm{CC}=\mathrm{CC}(\mathrm{N})=\mathrm{O}$ & $2.02 \mathrm{E}-07(\mathrm{~N})$ & 6.91E-06 \\
\hline 100.071332 & 1379.97 & 5 & 9 & 1 & 1 & 4.91 & $\mathrm{NC}(\mathrm{C}=\mathrm{CCC})=\mathrm{O}$ & $2.52 \mathrm{E}-06(\mathrm{~N})$ & $3.12 \mathrm{E}-07$ \\
\hline 101.022218 & 2932.23 & 4 & 4 & 0 & 3 & 1.65 & $\mathrm{O}=\mathrm{CC}=\mathrm{CC}(\mathrm{O})=\mathrm{O}$ & 2.01E-05 (E) & $8.30 \mathrm{E}-08$ \\
\hline 101.051638 & 1499.95 & 5 & 8 & 0 & 2 & 8.62 & $\mathrm{O}=\mathrm{C}(\mathrm{C}) \mathrm{CCC}=\mathrm{O}$ & 2.02E-03 (E) & 4.22E-10 \\
\hline 102.054463 & 1439.00 & 4 & 7 & 1 & 2 & 1.54 & $\mathrm{NCC}=\mathrm{CC}(\mathrm{O})=\mathrm{O}$ & $2.92 \mathrm{E}-05(\mathrm{~N})$ & $2.81 \mathrm{E}-08$ \\
\hline 102.089947 & 2487.60 & 5 & 11 & 1 & 1 & 1.94 & $\mathrm{NC}(\mathrm{CCCC})=\mathrm{O}$ & $3.50 \mathrm{E}-06(\mathrm{~N})$ & 4.05E-07 \\
\hline 103.03847 & 1574.86 & 4 & 6 & 0 & 3 & 1.05 & $\mathrm{CC}(\mathrm{C}(\mathrm{C}=\mathrm{O}) \mathrm{O})=\mathrm{O}$ & 2.00E-03 (E) & $4.48 \mathrm{E}-10$ \\
\hline 104.033168 & 2667.65 & 3 & 5 & 1 & 3 & 1.60 & $\mathrm{CC}=\mathrm{CON}(=\mathrm{O})=\mathrm{O}$ & $2.60 \mathrm{E}-02(\mathrm{E})$ & $5.85 \mathrm{E}-11$ \\
\hline 105.014648 & 2141.85 & 3 & 4 & 0 & 4 & 4.14 & $\mathrm{O}=\mathrm{C}(\mathrm{O}) \mathrm{C}(\mathrm{CO})=\mathrm{O}$ & 2.22E-06 (E) & $5.49 \mathrm{E}-07$ \\
\hline 109.096678 & 3357.59 & 8 & 12 & 0 & 0 & 5.05 & $\mathrm{C}=\mathrm{CC}=\mathrm{CC}=\mathrm{CCC}$ & 2.27E-02 (E) & $8.44 \mathrm{E}-11$ \\
\hline 110.058713 & 1424.19 & 6 & 7 & 1 & 1 & 1.88 & $\mathrm{OC} 1=\mathrm{CC}=\mathrm{CN}=\mathrm{C} 1 \mathrm{C}$ & $1.53 \mathrm{E}-03(\mathrm{~N})$ & $5.29 \mathrm{E}-10$ \\
\hline 111.043476 & 4684.00 & 6 & 6 & 0 & 2 & 1.13 & $\mathrm{O}=\mathrm{CC} 1=\mathrm{CC}=\mathrm{C}(\mathrm{O} 1) \mathrm{C}$ & $1.25 \mathrm{E}-03(\mathrm{~N})$ & 2.13E-09 \\
\hline 112.038821 & 2037.51 & 5 & 5 & 1 & 2 & 1.03 & $\mathrm{OC} 1=\mathrm{CC}(\mathrm{O})=\mathrm{CN}=\mathrm{C} 1$ & $5.32 \mathrm{E}-05(\mathrm{~N})$ & $2.18 \mathrm{E}-08$ \\
\hline 113.026161 & 2657.15 & 5 & 4 & 0 & 3 & -2.29 & $\mathrm{O}=\mathrm{C} 1 \mathrm{C}(\mathrm{C})=\mathrm{CC}(\mathrm{O} 1)=\mathrm{O}$ & 7.03E-04 (E) & 2.15E-09 \\
\hline 113.05728 & 3740.34 & 6 & 8 & 0 & 2 & 2.97 & $\mathrm{O}=\mathrm{CCCC}=\mathrm{CC}=\mathrm{O}$ & 4.92E-04 (E) & 4.33E-09 \\
\hline 114.055338 & 2438.47 & 5 & 7 & 1 & 2 & 0.17 & $\mathrm{C}=\mathrm{C}(\mathrm{C}=\mathrm{CC}(\mathrm{O})=\mathrm{O}) \mathrm{N}$ & $1.45 \mathrm{E}-05(\mathrm{~N})$ & $9.56 \mathrm{E}-08$ \\
\hline 115.038947 & 7677.84 & 5 & 6 & 0 & 3 & 0.57 & $\mathrm{O}=\mathrm{C}(\mathrm{C}) \mathrm{C}=\mathrm{CC}(\mathrm{O})=\mathrm{O}$ & 6.57E-06 (E) & $6.65 \mathrm{E}-07$ \\
\hline 116.033084 & 1330.37 & 4 & 5 & 1 & 3 & 1.68 & $\mathrm{C}=\mathrm{CC}=\mathrm{CON}(=\mathrm{O})=\mathrm{O}$ & $8.50 \mathrm{E}-03(\mathrm{E})$ & 8.92E-11 \\
\hline 116.064614 & 1348.08 & 5 & 9 & 1 & 2 & 6.54 & $\mathrm{CC}(\mathrm{N}) \mathrm{C}=\mathrm{CC}(\mathrm{O})=\mathrm{O}$ & $1.87 \mathrm{E}-05(\mathrm{~N})$ & 4.12E-08 \\
\hline 117.050497 & 2483.76 & 5 & 8 & 0 & 3 & 4.67 & $\mathrm{O}=\mathrm{C}(\mathrm{C}) \mathrm{CCC}(\mathrm{O})=\mathrm{O}$ & $1.00 \mathrm{E}-05(\mathrm{E})$ & $1.41 \mathrm{E}-07$ \\
\hline 118.046909 & 1887.46 & 4 & 7 & 1 & 3 & 3.51 & $\mathrm{CCC}=\mathrm{CON}(=\mathrm{O})=\mathrm{O}$ & $8.50 \mathrm{E}-03$ (E) & $1.27 \mathrm{E}-10$ \\
\hline 123.046863 & 1686.79 & 7 & 6 & 0 & 2 & -2.26 & $\mathrm{CC} 1=\mathrm{CC}(\mathrm{C}=\mathrm{CC} 1=\mathrm{O})=\mathrm{O}$ & $8.12 \mathrm{E}-06(\mathrm{E})$ & $1.18 \mathrm{E}-07$ \\
\hline 125.06148 & 1442.25 & 7 & 8 & 0 & 2 & -1.23 & $\mathrm{CC} 1=\mathrm{CC}=\mathrm{CC}(\mathrm{O})=\mathrm{C} 1 \mathrm{O}$ & $6.77 \mathrm{E}-06(\mathrm{~N})$ & $1.21 \mathrm{E}-07$ \\
\hline 126.051919 & 2178.90 & 6 & 7 & 1 & 2 & 3.58 & $\mathrm{OC} 1=\mathrm{CN}=\mathrm{C}(\mathrm{C}) \mathrm{C}(\mathrm{O})=\mathrm{C} 1$ & $1.36 \mathrm{E}-05(\mathrm{~N})$ & $9.10 \mathrm{E}-08$ \\
\hline 127.039667 & 4187.31 & 6 & 6 & 0 & 3 & -0.15 & $\mathrm{O}=\mathrm{C}(\mathrm{C}) \mathrm{C}=\mathrm{CC}(\mathrm{C}=\mathrm{O})=\mathrm{O}$ & 1.64E-04 (E) & $1.46 \mathrm{E}-08$ \\
\hline 127.112254 & 3174.50 & 8 & 14 & 0 & 1 & 0.04 & $\mathrm{O}=\mathrm{CCCCCC}=\mathrm{CC}$ & $1.45 \mathrm{E}-03(\mathrm{E})$ & $1.25 \mathrm{E}-09$ \\
\hline 128.03127 & 2068.51 & 5 & 5 & 1 & 3 & 3.50 & $\mathrm{O}=\mathrm{N}(\mathrm{C} 1=\mathrm{CC}=\mathrm{C}(\mathrm{C}) \mathrm{O} 1)=\mathrm{O}$ & $1.93 \mathrm{E}-03(\mathrm{~N})$ & $6.09 \mathrm{E}-10$ \\
\hline 129.053327 & 3905.59 & 6 & 8 & 0 & 3 & 1.84 & $\mathrm{O}=\mathrm{C}(\mathrm{C}=\mathrm{CC}(\mathrm{O}) \mathrm{C}=\mathrm{O}) \mathrm{C}$ & 6.36E-05 (E) & $3.50 \mathrm{E}-08$ \\
\hline
\end{tabular}


Table S8: DART-MS data from high-NO toluene oxidation (experiment 14).

\begin{tabular}{|c|c|c|c|c|c|c|c|c|c|}
\hline $\mathrm{m} / \mathrm{z}(+)(\mathrm{Da})$ & Intensity $^{a}$ & $\mathrm{C}$ & $\mathrm{H}$ & $\mathrm{N}$ & $\mathrm{O}$ & $\Delta^{b}(\mathrm{mDa})$ & Smiles & Est. VP $(\mathrm{atm})^{c}$ & Abundance $\left(\mathrm{R}_{f}\right)$ \\
\hline 130.052743 & 1472.64 & 5 & 7 & 1 & 3 & -2.32 & $\mathrm{O}=\mathrm{N}(\mathrm{OC}=\mathrm{CC}=\mathrm{CC})=\mathrm{O}$ & $2.78 \mathrm{E}-03(\mathrm{E})$ & $3.02 \mathrm{E}-10$ \\
\hline 131.035911 & 1919.85 & 5 & 6 & 0 & 4 & -1.48 & $\mathrm{OC}(\mathrm{C}(\mathrm{O})=\mathrm{C}(\mathrm{C}=\mathrm{O}) \mathrm{C})=\mathrm{O}$ & 1.09E-07 (E) & $1.00 \mathrm{E}-05$ \\
\hline 131.062715 & 1156.33 & 6 & 10 & 0 & 3 & 8.10 & $\mathrm{O}=\mathrm{C}(\mathrm{C}) \mathrm{CCC}(\mathrm{C}=\mathrm{O}) \mathrm{O}$ & 4.99E-05 (E) & $1.32 \mathrm{E}-08$ \\
\hline 133.047111 & 1311.02 & 5 & 8 & 0 & 4 & 2.97 & $\mathrm{OC}(\mathrm{C}(\mathrm{C}(\mathrm{C}) \mathrm{C}=\mathrm{O}) \mathrm{O})=\mathrm{O}$ & $1.22 \mathrm{E}-01(\mathrm{E})$ & $6.13 \mathrm{E}-12$ \\
\hline 138.049261 & 2335.43 & 7 & 7 & 1 & 2 & 6.24 & $\mathrm{CC} 1=\mathrm{C}(\mathrm{N}(=\mathrm{O})=\mathrm{O}) \mathrm{C}=\mathrm{CC}=\mathrm{C} 1$ & $2.18 \mathrm{E}-04(\mathrm{~N})$ & $6.12 \mathrm{E}-09$ \\
\hline 139.034538 & 2994.57 & 7 & 6 & 0 & 3 & 4.98 & $\mathrm{CC} 1=\mathrm{CC}(\mathrm{C}=\mathrm{C}(\mathrm{O}) \mathrm{C} 1=\mathrm{O})=\mathrm{O}$ & 5.26E-07 (E) & $3.24 \mathrm{E}-06$ \\
\hline 140.030245 & 2170.62 & 6 & 5 & 1 & 3 & 4.52 & $\mathrm{OC} 1=\mathrm{CC}=\mathrm{CC}=\mathrm{C} 1 \mathrm{~N}(=\mathrm{O})=\mathrm{O}$ & $8.71 \mathrm{E}-05(\mathrm{~N})$ & $1.42 \mathrm{E}-08$ \\
\hline 141.050361 & 5078.50 & 7 & 8 & 0 & 3 & 4.81 & $\mathrm{CC} 1=\mathrm{CC}=\mathrm{C}(\mathrm{O}) \mathrm{C}(\mathrm{O})=\mathrm{C} 1 \mathrm{O}$ & $5.97 \mathrm{E}-08(\mathrm{~N})$ & 4.85E-05 \\
\hline 142.046271 & 3022.13 & 6 & 7 & 1 & 3 & 4.15 & $\mathrm{C}=\mathrm{CC}=\mathrm{CC}(\mathrm{ON}(=\mathrm{O})=\mathrm{O})=\mathrm{C}$ & 9.08E-04 (E) & $1.90 \mathrm{E}-09$ \\
\hline 143.031682 & 2933.82 & 6 & 6 & 0 & 4 & 2.75 & $\mathrm{O}=\mathrm{C}(\mathrm{C}) \mathrm{C}=\mathrm{CC}(\mathrm{C}(\mathrm{O})=\mathrm{O})=\mathrm{O}$ & 5.77E-07 (E) & $2.90 \mathrm{E}-06$ \\
\hline 145.047972 & 2291.30 & 6 & 8 & 0 & 4 & 2.11 & $\mathrm{O}=\mathrm{C}(\mathrm{C}=\mathrm{CC}(\mathrm{O}) \mathrm{C}(\mathrm{O})=\mathrm{O}) \mathrm{C}$ & 3.56E-08 (E) & $3.66 \mathrm{E}-05$ \\
\hline 152.068107 & 1146.80 & 8 & 9 & 1 & 2 & 3.05 & $\mathrm{NC}(\mathrm{C}=\mathrm{CC}=\mathrm{CC}=\mathrm{CC}=\mathrm{O})=\mathrm{O}$ & 4.05E-09 (N) & $1.61 \mathrm{E}-04$ \\
\hline 154.045164 & 2966.87 & 7 & 7 & 1 & 3 & 5.25 & $\mathrm{OC} 1=\mathrm{C}(\mathrm{N}(=\mathrm{O})=\mathrm{O}) \mathrm{C}=\mathrm{CC}=\mathrm{C} 1 \mathrm{C}$ & $1.77 \mathrm{E}-05(\mathrm{~N})$ & $9.55 \mathrm{E}-08$ \\
\hline 155.034837 & 3800.38 & 7 & 6 & 0 & 4 & -0.40 & $\mathrm{CC} 1=\mathrm{CC}(\mathrm{C}(\mathrm{O})=\mathrm{C}(\mathrm{O}) \mathrm{C} 1=\mathrm{O})=\mathrm{O}$ & 8.26E-09 (E) & 2.62E-04 \\
\hline 156.06424 & 1417.63 & 7 & 9 & 1 & 3 & 1.83 & $\mathrm{CC}=\mathrm{CC}=\mathrm{CC}=\mathrm{CON}(=\mathrm{O})=\mathrm{O}$ & 2.97E-04 (E) & 2.72E-09 \\
\hline 157.045695 & 5036.75 & 7 & 8 & 0 & 4 & 4.39 & $\mathrm{CC} 1=\mathrm{CC}(\mathrm{O})=\mathrm{C}(\mathrm{O}) \mathrm{C}(\mathrm{O})=\mathrm{C} 1 \mathrm{O}$ & $3.28 \mathrm{E}-10(\mathrm{~N})$ & $8.74 \mathrm{E}-03$ \\
\hline 158.044945 & 1517.74 & 6 & 7 & 1 & 4 & 0.39 & $\mathrm{O}=\mathrm{N}(\mathrm{OC}=\mathrm{CC}(\mathrm{CC}=\mathrm{C})=\mathrm{O})=\mathrm{O}$ & 3.80E-05 (E) & 2.27E-08 \\
\hline 159.062128 & 1321.64 & 7 & 10 & 0 & 4 & 3.61 & $\mathrm{CC}(\mathrm{C}=\mathrm{CC}(\mathrm{C}(\mathrm{O}) \mathrm{C}=\mathrm{O}) \mathrm{O})=\mathrm{O}$ & 3.41E-07 (E) & $2.21 \mathrm{E}-06$ \\
\hline 161.046849 & 1846.47 & 6 & 8 & 0 & 5 & -1.85 & $\mathrm{O}=\mathrm{C}(\mathrm{CO}) \mathrm{C}=\mathrm{CC}(\mathrm{C}(\mathrm{O})=\mathrm{O}) \mathrm{O}$ & $1.79 \mathrm{E}-10(\mathrm{E})$ & $5.89 \mathrm{E}-03$ \\
\hline 170.046401 & 1573.59 & 7 & 7 & 1 & 4 & -1.07 & $\begin{array}{l}\mathrm{OC} 1=\mathrm{C}(\mathrm{O}) \mathrm{C}(\mathrm{N}(=\mathrm{O})=\mathrm{O}) \\
=\mathrm{CC}=\mathrm{C} 1 \mathrm{C}\end{array}$ & 2.01E-07 (N) & 4.46E-06 \\
\hline 173.044149 & 1855.62 & 7 & 8 & 0 & 5 & 0.85 & $\begin{array}{l}\mathrm{CC} 1=\mathrm{C}(\mathrm{O}) \mathrm{C}(\mathrm{O}) \\
=\mathrm{C}(\mathrm{C}(\mathrm{O})=\mathrm{C} 1 \mathrm{O}) \mathrm{O}\end{array}$ & $1.12 \mathrm{E}-12(\mathrm{~N})$ & $9.44 \mathrm{E}-01$ \\
\hline 174.069813 & 1324.07 & 7 & 11 & 1 & 4 & 6.82 & $\mathrm{O}=\mathrm{N}(\mathrm{OC}=\mathrm{CCCCCC}=\mathrm{O})=\mathrm{O}$ & $1.90 \mathrm{E}-05(\mathrm{E})$ & $3.98 \mathrm{E}-08$ \\
\hline 175.059781 & 1319.16 & 7 & 10 & 0 & 5 & 0.87 & $\begin{array}{l}\mathrm{O}=\mathrm{C}(\mathrm{C}) \mathrm{C}=\mathrm{CC}(\mathrm{O}) \mathrm{C}(\mathrm{O}) \\
\mathrm{C}(\mathrm{O})=\mathrm{O}\end{array}$ & $1.33 \mathrm{E}-10(\mathrm{E})$ & $5.64 \mathrm{E}-03$ \\
\hline 177.157199 & 1399.51 & 9 & 20 & 0 & 3 & -8.13 & $\operatorname{CCCCC}(\mathrm{O}) \mathrm{C}(\mathrm{O}) \mathrm{CCCO}$ & 2.29E-09 (E) & $3.49 \mathrm{E}-04$ \\
\hline 178.069959 & 2175.62 & 6 & 11 & 1 & 5 & 1.59 & $\mathrm{O}=\mathrm{N}(\mathrm{OCCCCCC}(\mathrm{O})=\mathrm{O})=\mathrm{O}$ & $2.20 \mathrm{E}-07(\mathrm{E})$ & 5.63E-06 \\
\hline 223.064145 & 2037.80 & 11 & 10 & 0 & 5 & -3.50 & $\begin{array}{l}\mathrm{O}=\mathrm{C}(\mathrm{O}) \mathrm{C}=\mathrm{CC}=\mathrm{CC}=\mathrm{CC} \\
=\mathrm{CC}(\mathrm{C}(\mathrm{O})=\mathrm{O})=\mathrm{O}\end{array}$ & 3.35E-11 (E) & $3.46 \mathrm{E}-02$ \\
\hline
\end{tabular}

${ }^{a}$ (A.U.) ${ }^{b}$ The difference between the measured and proposed compound exact mass. ${ }^{b}$ Est. VP $=$ Estimated vapor pressure.

Estimation Method in parenthesis: $\mathrm{E}=$ EVAPORATION method, and $\mathrm{N}=$ Nannoolal method.

Other studies have reported structural isomers of the compounds listed in Table S6, S7, and S8 in the gas-phase and particlephase from toluene SOA (Jang and Kamens, 2001; Sato et al., 2007). As here, peaks for $\mathrm{C}_{7} \mathrm{H}_{8} \mathrm{O}_{4}$ and $\mathrm{C}_{7} \mathrm{H}_{8} \mathrm{O}_{5}$ had the largest 


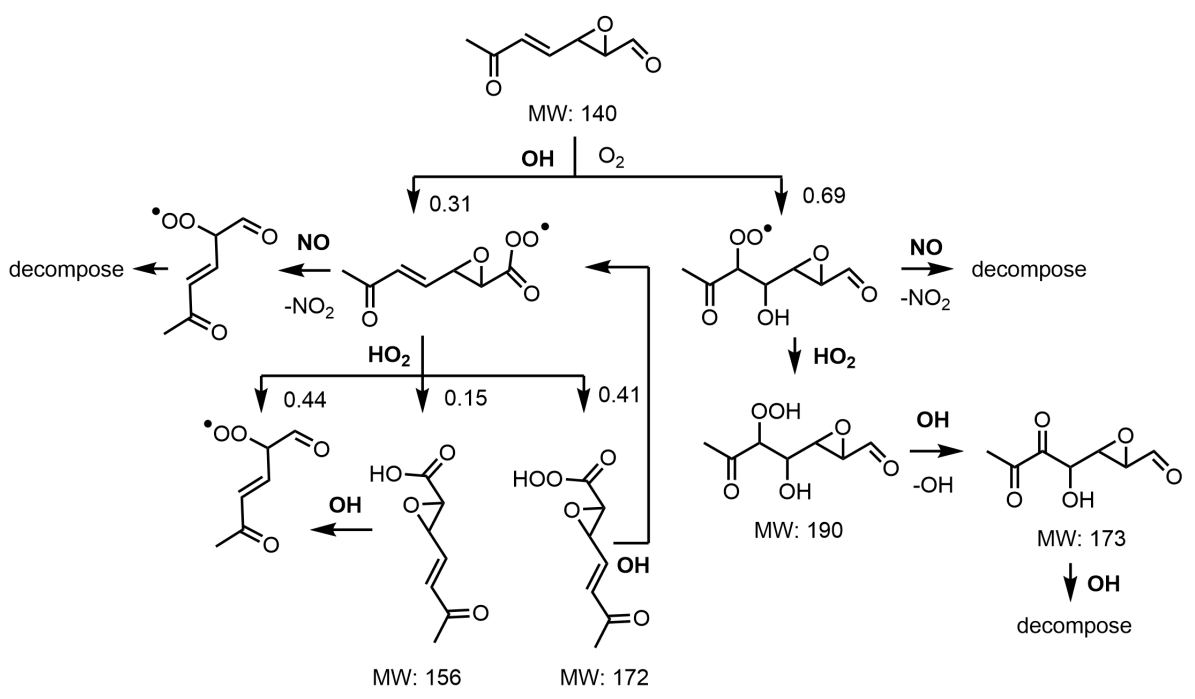

Figure S6. Epoxide pathway oxidation mechanism under both low- and high-NO conditions as recommended by MCM v3.3.1

intensity in the particle-phase measurements in the study by Sato et al. (2007), but it should be noted that only $1 \%$ of the SOA constituents were quantified in that study. Both of these studies suggest that the compounds are ring-opening products not produced from the cresol pathway. Given the new evidence from the $\mathrm{CF}_{3} \mathrm{O}^{-} \mathrm{CIMS}$ in this study, it is clear that these compounds are produced from the cresol pathway.

$5 \quad$ Products detected in the particle-phase by the DART-MS under $o$-cresol high NO conditions are shown in Figure S7. An oligomer product, $\mathrm{C}_{15} \mathrm{H}_{22} \mathrm{O}_{4}$, is detected as one of the dominant products in $o$-cresol oxidation under high-NO conditions (Figure S7). It is possible this product forms from oligomerization of trihydroxy toluene and $\mathrm{C}_{8} \mathrm{H}_{14} \mathrm{O}$ to form a hemiacetal. 


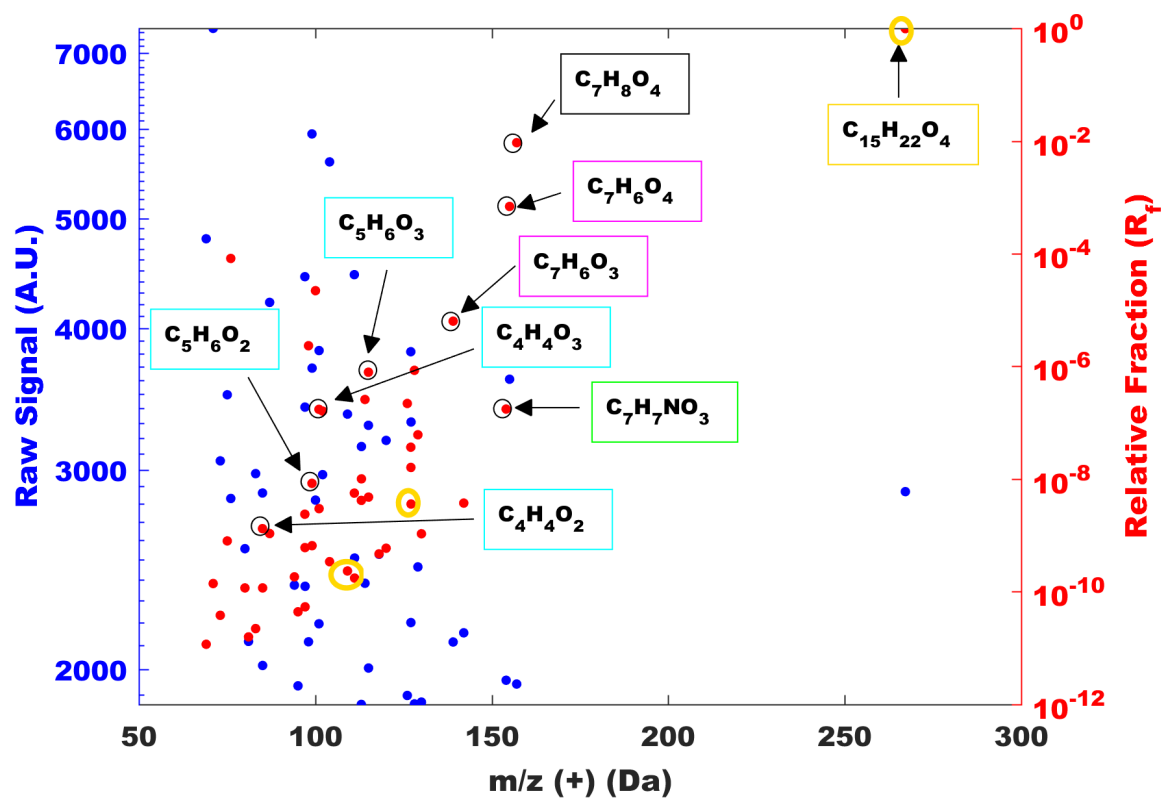

Figure S7. Products detected by DART-MS in the particle phase during oxidation of $o$-cresol under high NO conditions (experiment 15 ) with boxes identifying the following types of compounds: polyols (black), methyl benzoquinone type compounds (magenta), decomposition products from the bicyclic intermediate pathway (cyan), products with more than 7 carbons (gold), and nitro compounds (green). 


\section{References}

Bardini, P.: Atmospheric chemistry of dimethylphenols and nitrophenols, Ph.D. Thesis, University College Cork, Thesis, 2006.

Bejan, I., Barnes, I., Olariu, R., Zhou, S., Wiesen, P., and Benter, T.: Investigations on the gas-phase photolysis and OH radical kinetics of methyl-2-nitrophenols, Phys. Chem. Chem. Phys., 9, 5686-5692, 2007.

5 Berho, F., Caralp, F., Rayez, M. T., Lesclaux, R., and Ratajczak, E.: Kinetics and thermochemistry of the reversible combination reaction of the phenoxy radical with NO, J. Phys. Chem. A., 102, 1-8, 1998.

Chen, J., Wenger, J. C., and Venables, D. S.: Near-ultraviolet absorption cross sections of nitrophenols and their potential influence on tropospheric oxidation capacity, J. Phys. Chem. A., 115, 12 235-12 242, 2011.

Dewar, M. J. S. and Stewart, J. J. P.: A new procedure for calculating molecular polarizabilities; Applications using MNDO., Chem. Phys. Lett., 111, 416-420, 1984.

Jang, M. and Kamens, R. M.: Characterization of secondary aerosol from the photooxiation of toluene in the presense of $\mathrm{NO}_{x}$ and 1-propene, Environ. Sci. Technol., 35, 3626-3639, 2001.

Klotz, B., Sorensen, S., Barnes, I., Becker, K. H., Etzkorn, T., Volkamer, R., Platt, U., Wirtz, K., and Martin-Reviejo, M.: Atmospheric oxidation of toluene in a large-volume outdoor photoreactor: in situ determination of ring-retaining product yields, J. Phys. Chem. A., $102,1998$.

Lide, D. R.: CRC Handbook of Chemistry and Physics, vol. 82, CRC Press LLC, Boca Raton, Florida, 2001.

McClellan, A. L.: Table of Experimental Dipole Moments, vol. 2, Rahara Enterprises, El Cerrito, Ca, 1974.

Olariu, R. I., Barnes, I., Becker, K. H., and Klotz, B.: Rate coefficients for the gas-phase reaction of OH radicals with selected dihydroxybenzenes and benzoquinones, Int. J. Chem. Kinet., 32, 696-702, 2000.

Olariu, R. I., Klotz, B., Barnes, I., Becker, K. H., and Mocanu, R.: FT-IR study of the ring-retaining products from the reaction of OH radicals with phenol, $o-, m$-, and $p$-cresol, Atmos. Environ., 36, 3685-3697, 2002.

Pedersen, T., Larsen, N. W., and Nygaard, L.: Microwave spectra of the six monodeuteriophenols. Molecular structure, dipole moment, and barrier to internal rotation of phenol., J. Mol. Structure, 4, 59-77, 1969.

Sato, K., Hatakeyama, S., and Imamura, T.: Secondary organic aerosol formation during the photooxidation of toluene: $\mathrm{NO}_{x}$ dependence of chemical composition, J. Phys. Chem. A., 111, 9796-9808, 2007.

$\mathrm{Su}$, T. and Chesnavich, W. J.: Parametrization of the ion-polar molecule collision rate constant by trajectory calculations, J. Chem. Phys., 76, 5183-5185, 1982.

Tao, Z. and Li, Z.: A kinetics study on reactions of $\mathrm{C}_{6} \mathrm{H}_{5} \mathrm{O}$ with $\mathrm{C}_{6} \mathrm{H}_{5} \mathrm{O}$ and $\mathrm{O}_{3}$ at $298 \mathrm{k}$, Int. J. Chem. Kinet., 31, 65-72, 1999.

Yu, T., Mebel, A. M., and Lin, M. C.: Reaction of phenoxy radical with nitric oxide, J. Phys. Org. Chem., 8, 47-53, 1995. 